\title{
Analysis Report
}

<Sample Information>

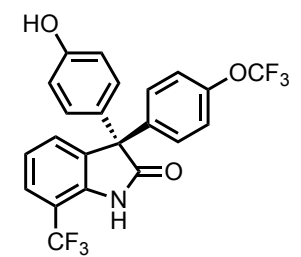

ErSO (1)

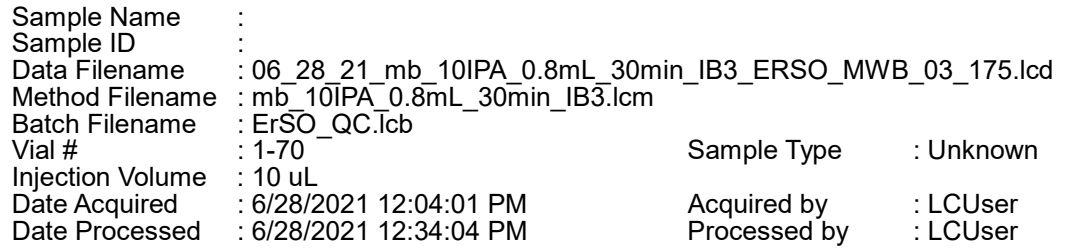

\section{$<$ Chromatogram>}

mAU

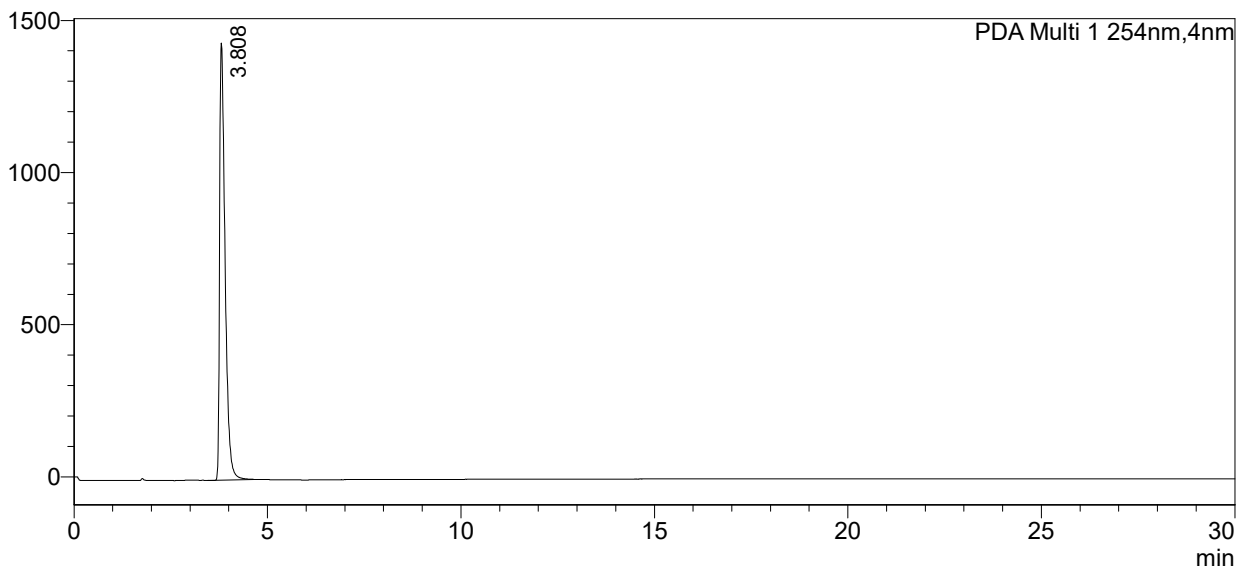

<Peak Table>

PDA Ch1 254nm

\begin{tabular}{|c|c|c|c|c|c|c|}
\hline Peak\# & Ret. Time & Area & Height & Conc. & Area\% & Height $\%$ \\
\hline 1 & 3.808 & 14151523 & 1436126 & 0.000 & 100.000 & 100.000 \\
\hline Total & & 14151523 & 1436126 & & 100.000 & 100.000 \\
\hline
\end{tabular}




\section{Chiral HPLC Trace Analysis Report}

\section{<Sample Information>}

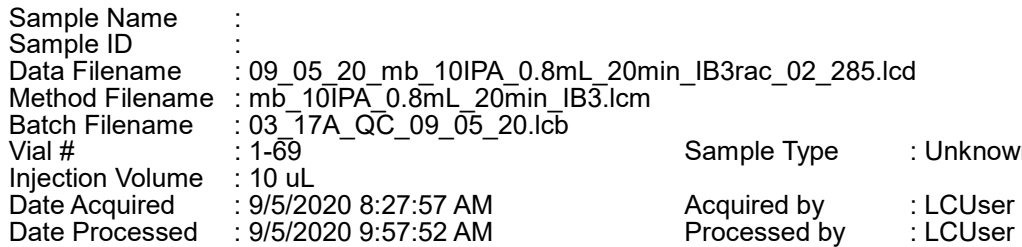

\section{$<$ Chromatogram>}

$\mathrm{mAU}$

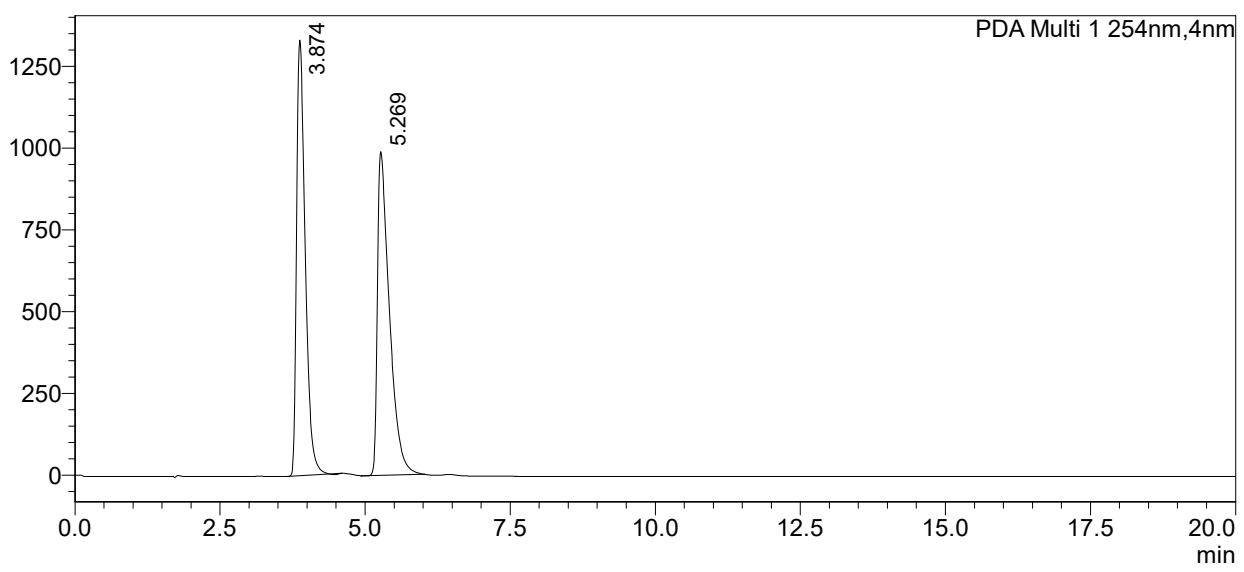

<Peak Table>

\begin{tabular}{|c|c|c|c|c|c|c|}
\hline \multicolumn{7}{|c|}{$54 \mathrm{~nm}$} \\
\hline 1 & 3.874 & 13801074 & 1332174 & 0.000 & 49.342 & 57.371 \\
\hline 2 & 5.269 & 14169318 & 989864 & 0.000 & 50.658 & 42.629 \\
\hline Total & & 27970393 & 2322038 & & 100.000 & 100.000 \\
\hline
\end{tabular}




\section{Chiral HPLC Trace}

\section{Analysis Report}

<Sample Information>

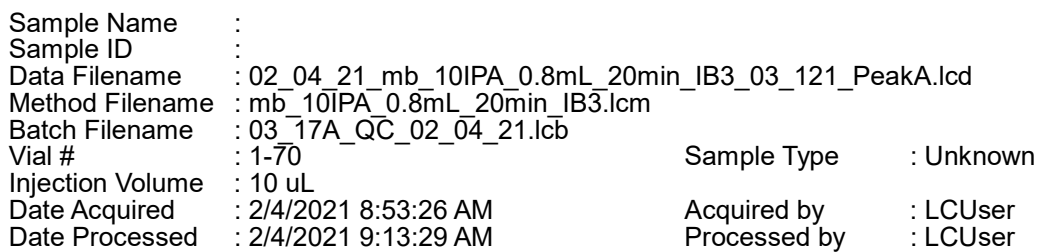

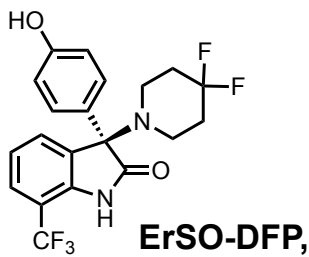

(R)-2

\section{$<$ Chromatogram $>$}

mAU

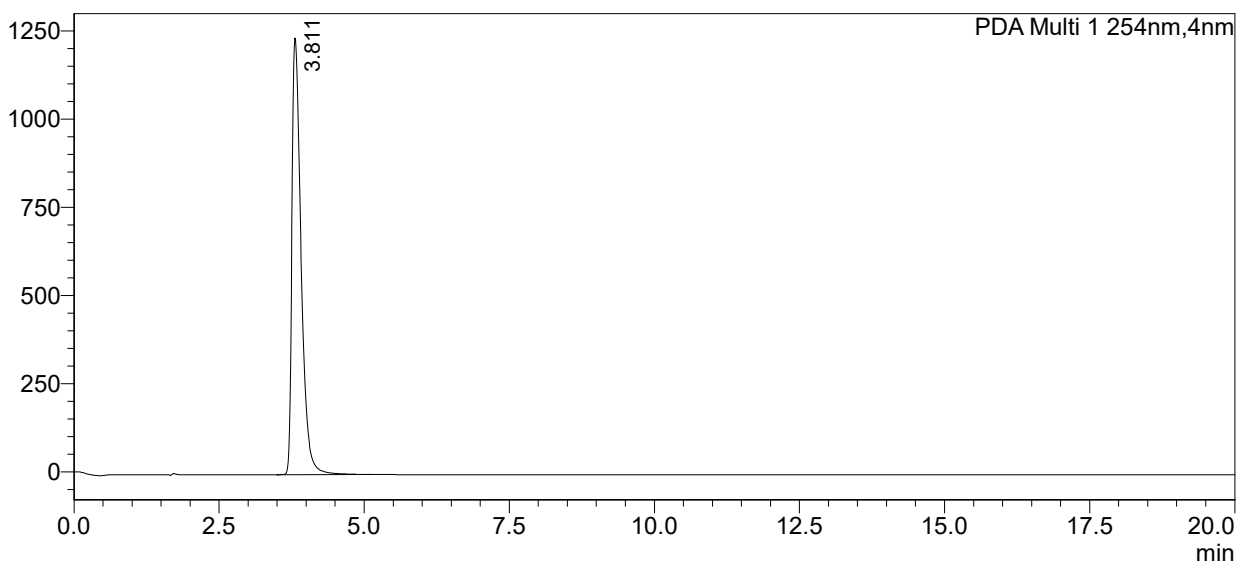

<Peak Table>

PDA Ch1 254nm

\begin{tabular}{|c|c|c|c|c|c|c|}
\hline Peak\# & Ret. Time & Area & Height & Conc. & Area\% & Height $\%$ \\
\hline 1 & 3.811 & 13663307 & 1237528 & 0.000 & 100.000 & 100.000 \\
\hline Total & & 13663307 & 1237528 & & 100.000 & 100.000 \\
\hline
\end{tabular}




\section{Analysis Report

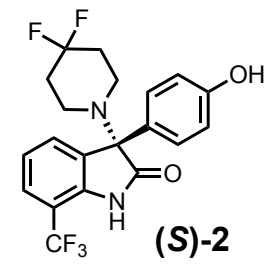

<Sample Information>

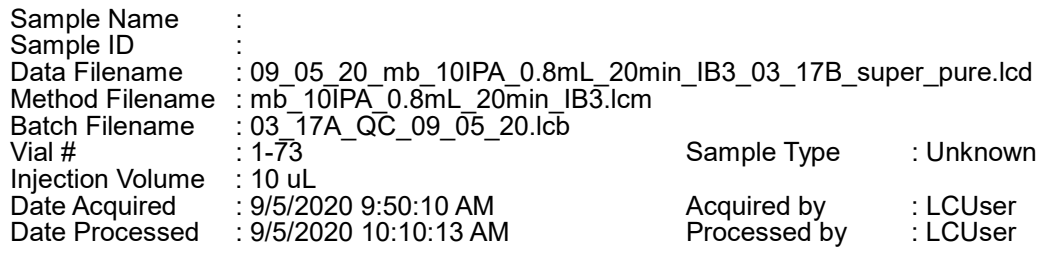

\section{$<$ Chromatogram $>$}

mAU

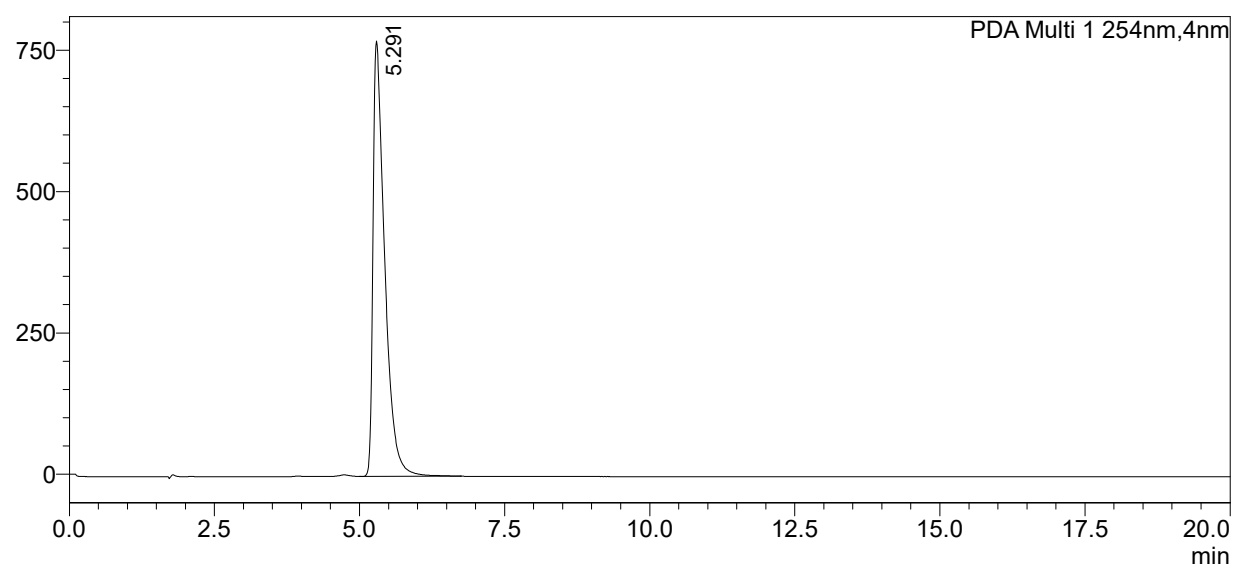

<Peak Table>

PDA Ch1 254nm

\begin{tabular}{|c|c|c|c|c|c|c|}
\hline Peak\# & Ret. Time & Area & Height & Conc. & Area\% & Height $\%$ \\
\hline 1 & 5.291 & 10785067 & 769908 & 0.000 & 100.000 & 100.000 \\
\hline Total & & 10785067 & 769908 & & 100.000 & 100.000 \\
\hline
\end{tabular}


Chiral HPLC Trace

Analysis Report

<Sample Information>

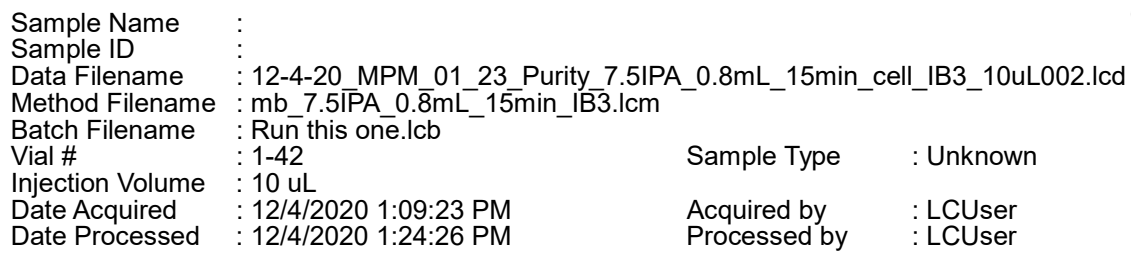

\section{$<$ Chromatogram $>$}

mAU

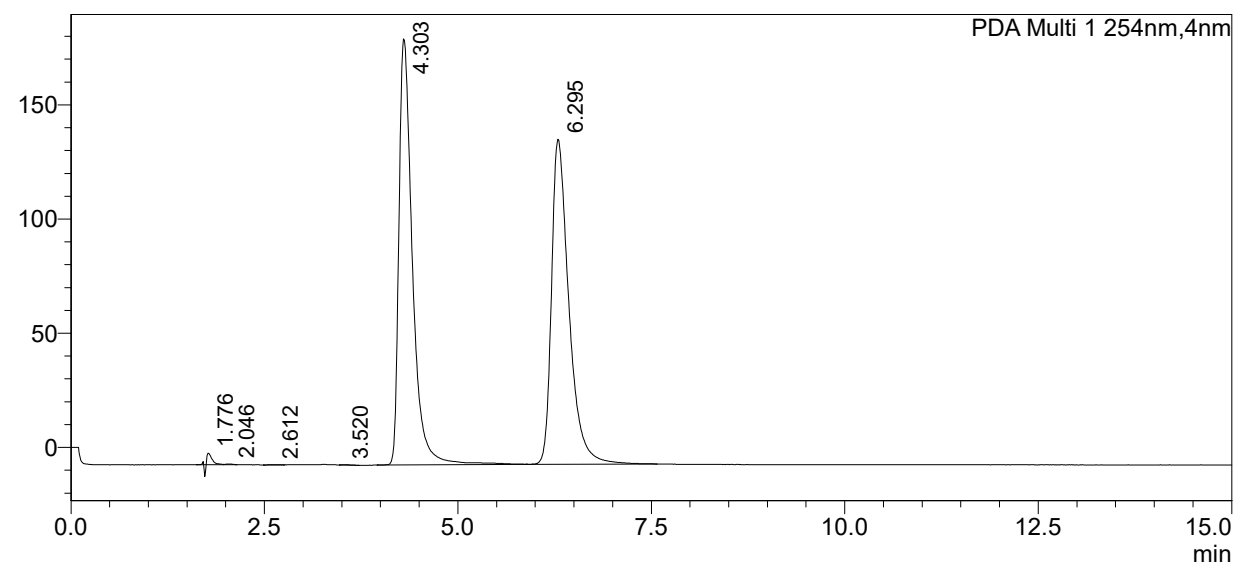

<Peak Table>

\begin{tabular}{|c|c|c|c|c|c|c|}
\hline \multicolumn{7}{|c|}{ PDA Ch1 254nm } \\
\hline Peak\# & Ret. Time & Area & Height & Conc. & Area $\%$ & Height $\%$ \\
\hline 1 & 1.776 & 19484 & 5036 & 0.000 & 0.430 & 1.504 \\
\hline 2 & 2.046 & 2536 & 391 & 0.000 & 0.056 & 0.117 \\
\hline 3 & 2.612 & 2270 & 206 & 0.000 & 0.050 & 0.061 \\
\hline 4 & 3.520 & 1014 & 114 & 0.000 & 0.022 & 0.034 \\
\hline 5 & 4.303 & 2273927 & 186594 & 0.000 & 50.235 & 55.738 \\
\hline 6 & 6.295 & 2227388 & 142426 & 0.000 & 49.206 & 42.545 \\
\hline Total & & 4526619 & 334767 & & 100.000 & 100.000 \\
\hline
\end{tabular}




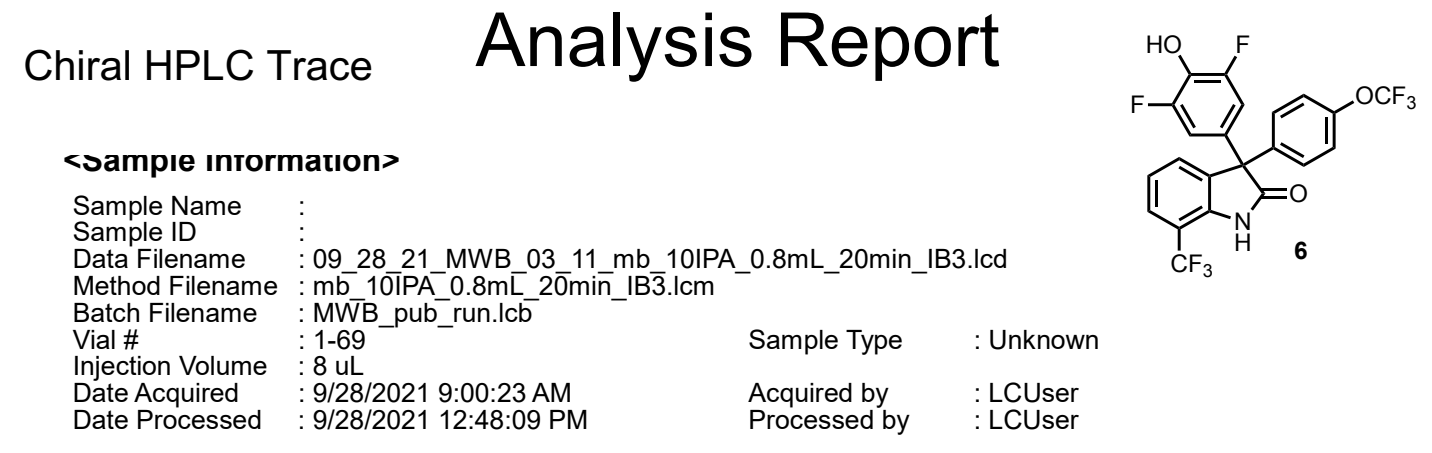

\section{<Chromatogram>}

mAU

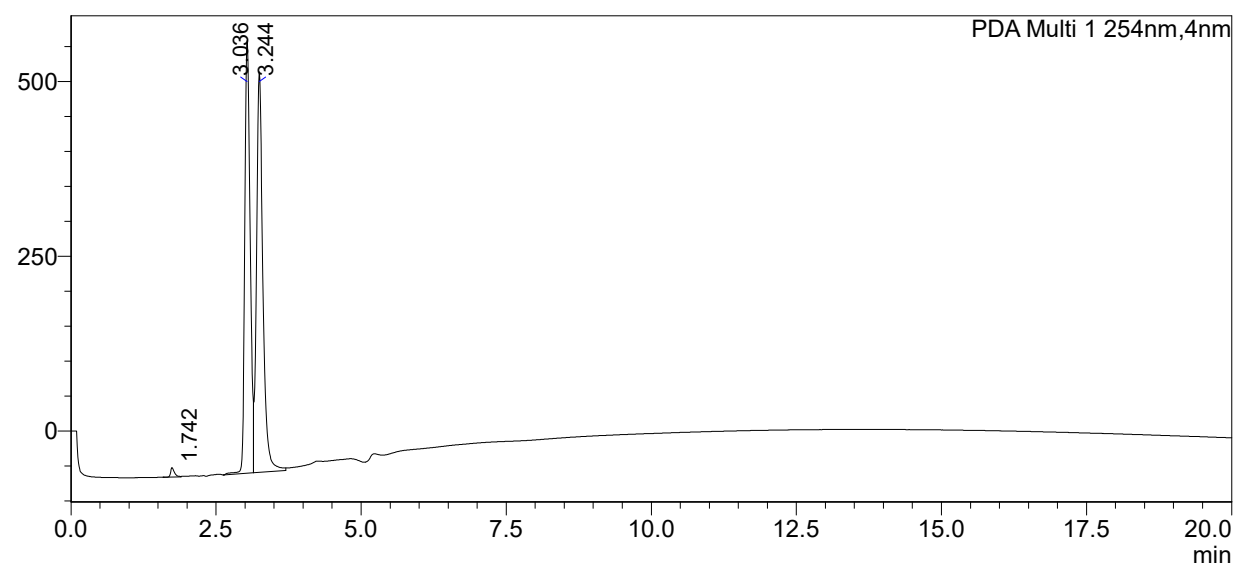

<Peak Table>

PDA Ch1 254nm

Peak\# Ret. Time

\begin{tabular}{|r|r|r|r|r|r|r|}
\hline Peak\# & Ret. Time & \multicolumn{1}{|c|}{ Area } & Height & Conc. & Area\% & \multicolumn{1}{c|}{ Height\% } \\
\hline 1 & 1.742 & 62758 & 13301 & 0.000 & 0.746 & 1.103 \\
\hline 2 & 3.036 & 3987990 & 619720 & 0.000 & 47.411 & 51.371 \\
\hline 3 & 3.244 & 4360817 & 573330 & 0.000 & 51.843 & 47.526 \\
\hline Total & & 8411564 & 1206351 & & 100.000 & 100.000 \\
\hline
\end{tabular}




\section{Chiral HPLC Trace Analysis Report}

<Sample Information>
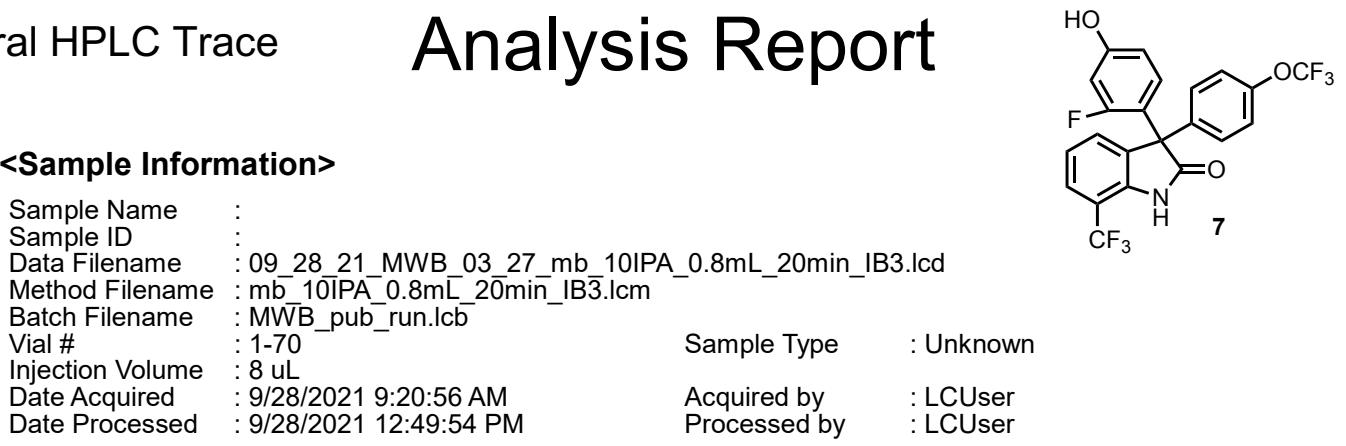

\section{$<$ Chromatogram $>$}

mAU

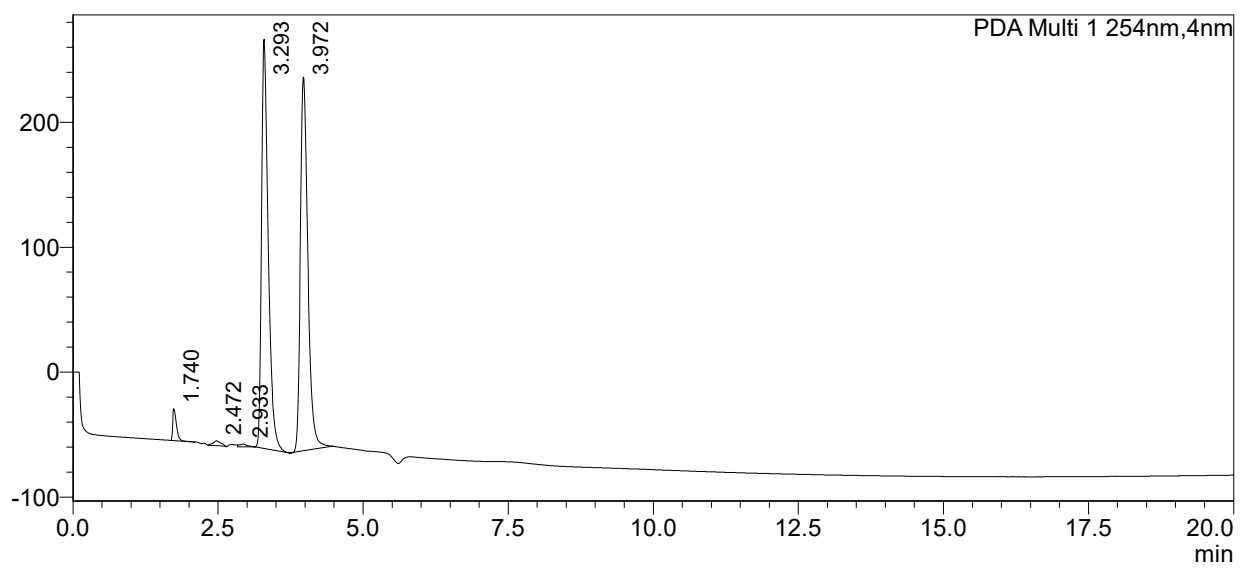

<Peak Table>

PDA Ch1 254nm

\begin{tabular}{|r|r|r|r|r|r|r|}
\hline Peak\# & Ret. Time & \multicolumn{1}{|c|}{ Area } & Height & Conc. & Area\% & Height $\%$ \\
\hline 1 & 1.740 & 113553 & 25529 & 0.000 & 2.065 & 3.879 \\
\hline 2 & 2.472 & 35621 & 3866 & 0.000 & 0.648 & 0.587 \\
\hline 3 & 2.933 & 18840 & 2138 & 0.000 & 0.343 & 0.325 \\
\hline 4 & 3.293 & 2651512 & 327422 & 0.000 & 48.226 & 49.753 \\
\hline 5 & 3.972 & 2678579 & 299142 & 0.000 & 48.718 & 45.456 \\
\hline Total & & 5498105 & 658097 & & 100.000 & 100.000 \\
\hline
\end{tabular}




\section{HPLC Trace}

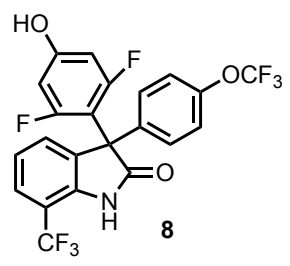

\section{Sample Purity - Detailed Report}

\section{Sequence Name:}

Data file:

Sample name:

Instrument:

Inj. volume:

Acq. method:

Processing method:
SingleSample

MPM_03_105P2.dx

MPM_03_105P2

$6125 \mathrm{C}$ LCMS

5.000

Measure_purity_esi-305.amx

Compound_Purity_MG_default.pmx

$\begin{array}{ll}\text { Project Name: } & \text { Default } \\ \text { Operator : } & \text { SYSTEM } \\ \text { Acquired on: } & \text { 2021-09-21 16:58:39-05:00 } \\ \text { Location: } & 31\end{array}$

Location:
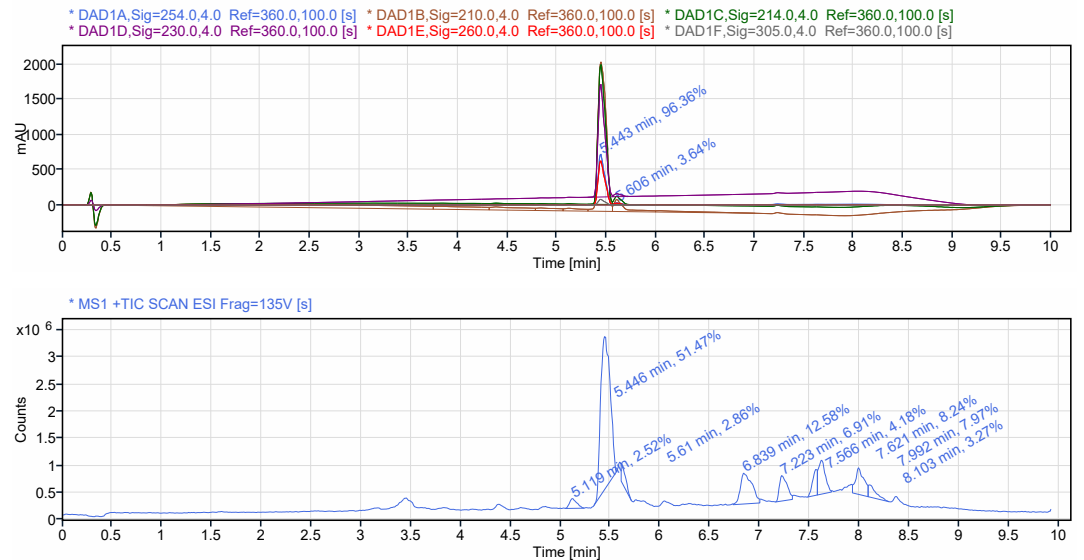

Peak Results (Area Percent at least 1\%)

RT (min) Signal Description

5.119 * MS1 +TIC SCAN ESI Frag $=135 \mathrm{~V}$ [s]

$5.443 \quad{ }^{*} \mathrm{DAD} 1 \mathrm{~A}, \mathrm{Sig}=254.0,4.0$ Ref=360.0,100.0 [s]

$5.446 \quad$ * MS1 +TIC SCAN ESI Frag $=135 \mathrm{~V}$ [s]

5.606 * DAD1A,Sig=254.0,4.0 Ref=360.0,100.0 [s]

$5.610 \quad{ }^{*} \mathrm{MS} 1+$ TIC SCAN ESI Frag $=135 \mathrm{~V}$ [s]

$6.839 *$ MS1 +TIC SCAN ESI Frag=135V [s]

$7.223{ }^{*} \mathrm{MS} 1+$ TIC SCAN ESI Frag $=135 \mathrm{~V}$ [s]

$7.566 \quad{ }^{*} \mathrm{MS} 1+$ TIC SCAN ESI Frag $=135 \mathrm{~V}$ [s]

$7.621 \quad$ * MS1 +TIC SCAN ESI Frag=135V [s]

$7.992{ }^{*}$ MS1 + TIC SCAN ESI Frag=135V [s]

$8.103 \quad{ }^{*}$ MS1 + TIC SCAN ESI Frag $=135 \mathrm{~V}$ [s]

$\begin{array}{rrrr}\text { Width }(\min ) & \text { Area } & \text { Height } & \text { Area\% } \\ 0.191 & 920062.5 & 180307.5 & 2.52 \\ 0.272 & 3470.9 & 720.5 & 96.36 \\ 0.225 & 18783354.7 & 2817456.1 & 51.47 \\ 0.247 & 131.2 & 27.0 & 3.64 \\ 0.104 & 1044272.3 & 330308.4 & 2.86 \\ 0.268 & 4591011.1 & 574736.8 & 12.58 \\ 0.170 & 2522262.5 & 469961.6 & 6.91 \\ 0.097 & 1525416.3 & 478167.2 & 4.18 \\ 0.141 & 3008220.1 & 628254.7 & 8.24 \\ 0.157 & 2907835.5 & 490966.0 & 7.97 \\ 0.211 & 1193867.6 & 223965.8 & 3.27\end{array}$




\section{Chiral HPLC Trace}

\section{Analysis Report}

\section{<Sample Information>}
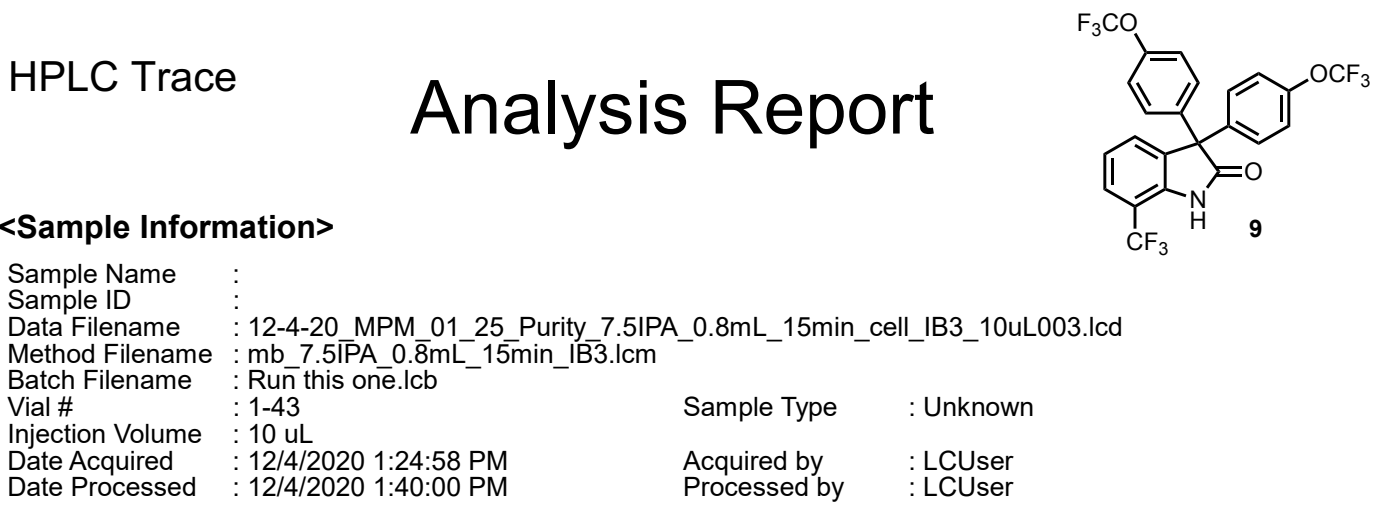

\section{<Chromatogram>}

mAU

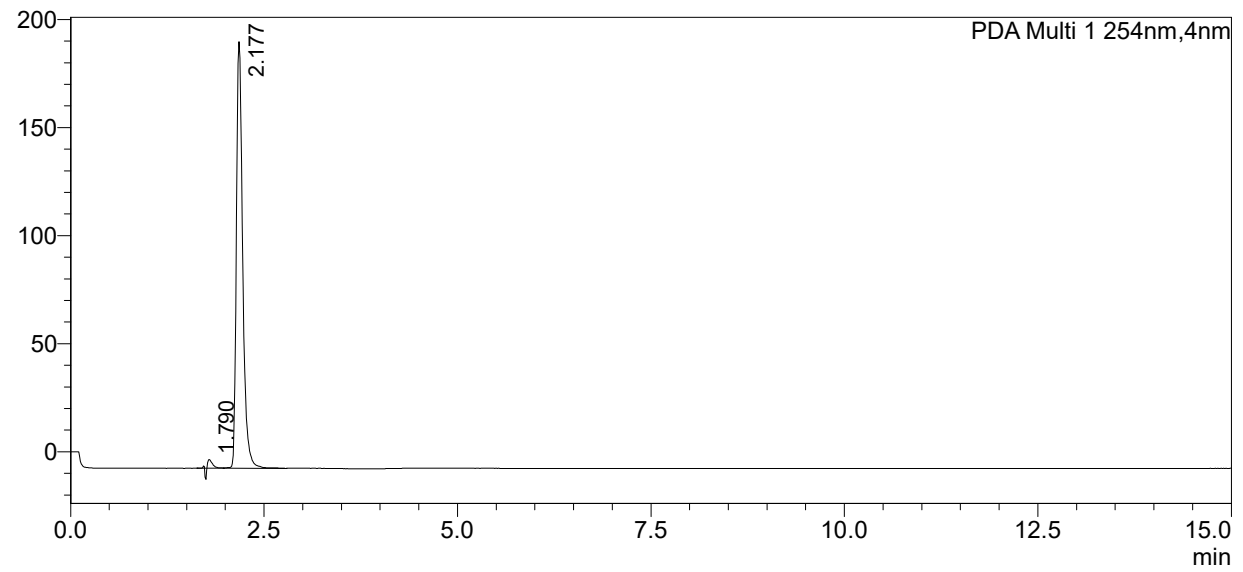

<Peak Table>

PDA Ch1 254nm

\begin{tabular}{|r|r|r|r|r|r|r|}
\hline Peak\# & Ret. Time & \multicolumn{1}{|c|}{ Area } & \multicolumn{1}{|c|}{ Height } & Conc. & Area\% & \multicolumn{1}{|c|}{ Height $\%$} \\
\hline 1 & 1.790 & 13043 & 4109 & 0.000 & 1.152 & 2.040 \\
\hline 2 & 2.177 & 1118942 & 197350 & 0.000 & 98.848 & 97.960 \\
\hline Total & & 1131985 & 201459 & & 100.000 & 100.000 \\
\hline
\end{tabular}




\section{Chiral HPLC Trace Analysis Report}

\section{<Sample Information>}
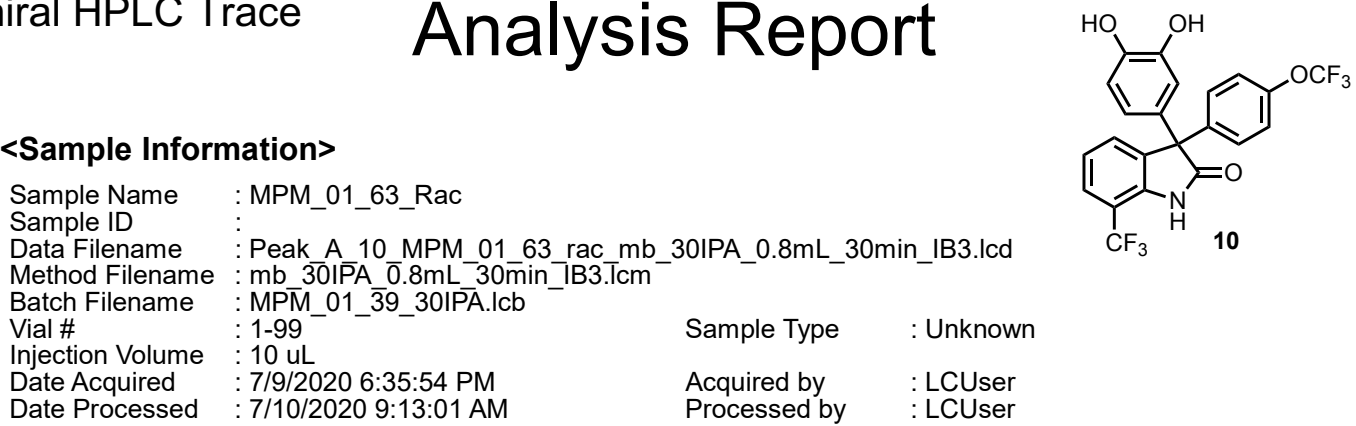

\section{$<$ Chromatogram $>$}

mAU

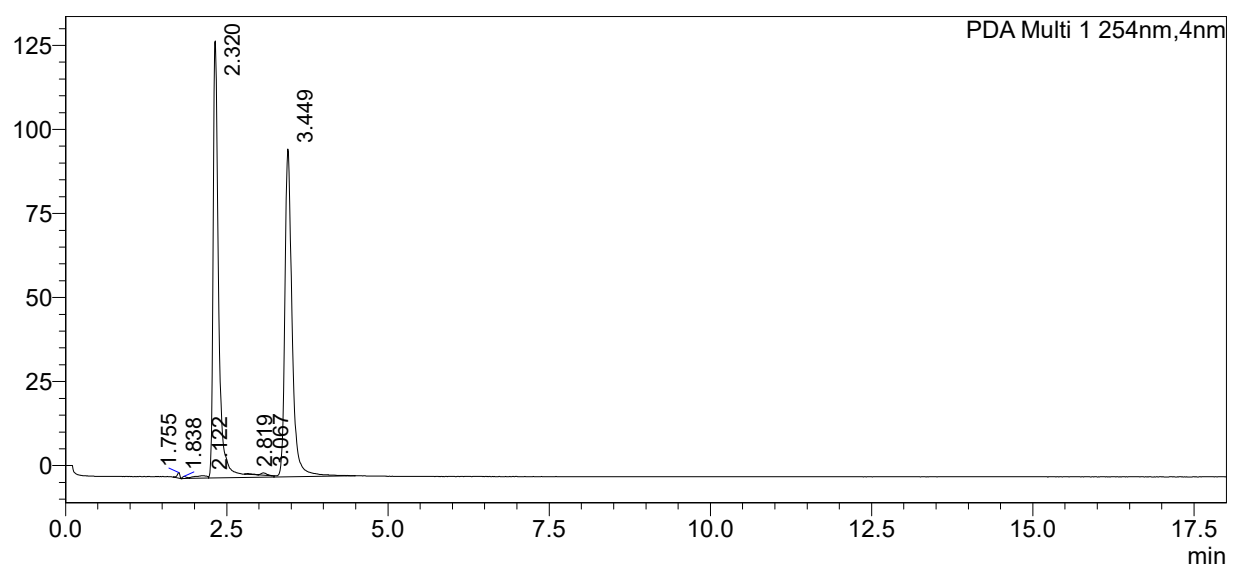

<Peak Table>

PDA Ch1 254nm

\begin{tabular}{|r|r|r|r|r|r|r|}
\hline Peak\# Ret. Time & \multicolumn{1}{|c|}{ Area } & Height & Conc. & Area\% & Height $\%$ \\
\hline 1 & 1.755 & 4369 & 1691 & 0.000 & 0.279 & 0.731 \\
\hline 2 & 1.838 & 2816 & 605 & 0.000 & 0.180 & 0.262 \\
\hline 3 & 2.122 & 9850 & 733 & 0.000 & 0.630 & 0.317 \\
\hline 4 & 2.320 & 782333 & 129984 & 0.000 & 50.003 & 56.186 \\
\hline 5 & 2.819 & 1405 & 177 & 0.000 & 0.090 & 0.077 \\
\hline 6 & 3.067 & 4093 & 668 & 0.000 & 0.262 & 0.289 \\
\hline 7 & 3.449 & 759710 & 97486 & 0.000 & 48.557 & 42.139 \\
\hline Total & & 1564577 & 231345 & & 100.000 & 100.000 \\
\hline
\end{tabular}




\section{Chiral HPLC Trace Analysis Report}

<Sample Information>
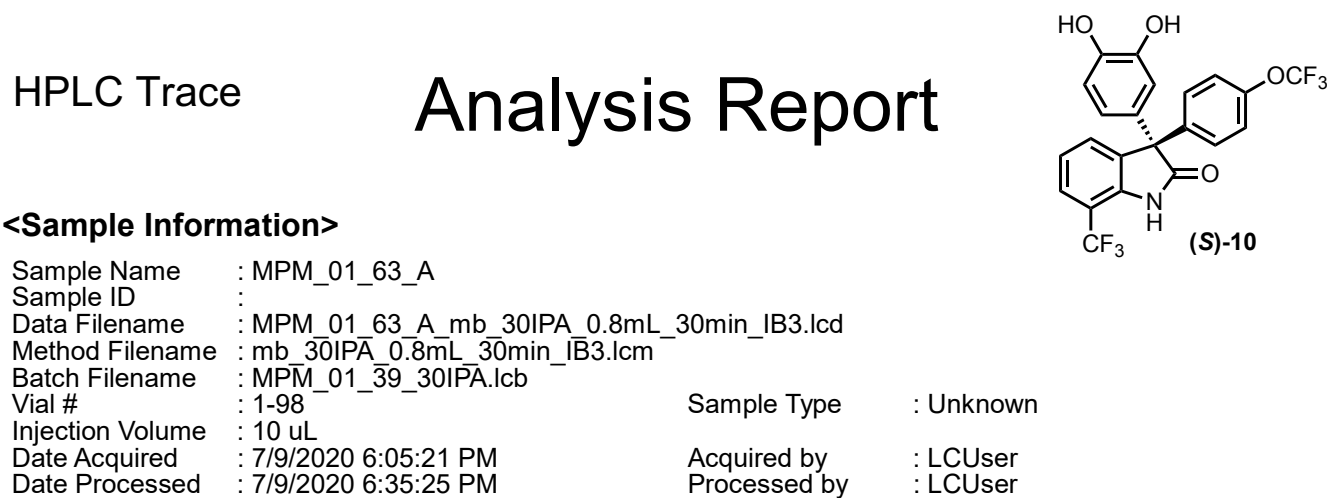

\section{$<$ Chromatogram $>$}

mAU

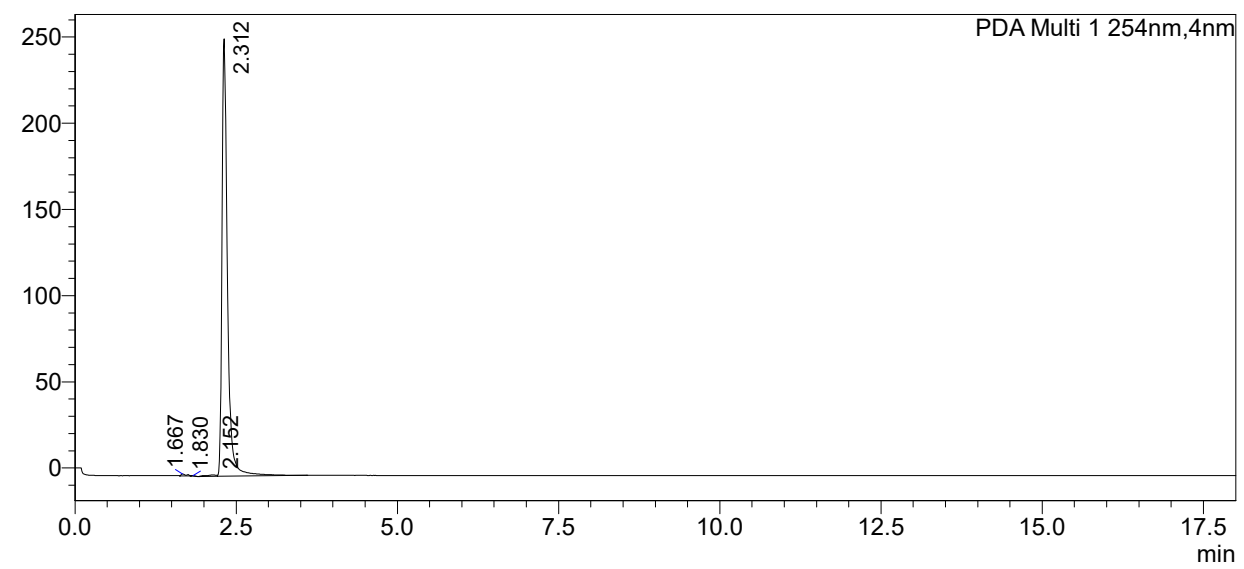

<Peak Table>

PDA Ch1 254nm

\begin{tabular}{|r|r|r|r|r|r|r|}
\hline Peak\# & Ret. Time & \multicolumn{1}{|c|}{ Area } & Height & \multicolumn{1}{c|}{ Conc. } & Area\% & Height\% \\
\hline 1 & 1.667 & 5689 & 1044 & 0.000 & 0.373 & 0.408 \\
\hline 2 & 1.830 & 1120 & 365 & 0.000 & 0.073 & 0.143 \\
\hline 3 & 2.152 & 9074 & 749 & 0.000 & 0.595 & 0.293 \\
\hline 4 & 2.312 & 1509407 & 253622 & 0.000 & 98.959 & 99.156 \\
\hline Total & & 1525290 & 255780 & & 100.000 & 100.000 \\
\hline
\end{tabular}




\section{Chiral HPLC Trace Analysis Report}

<Sample Information>
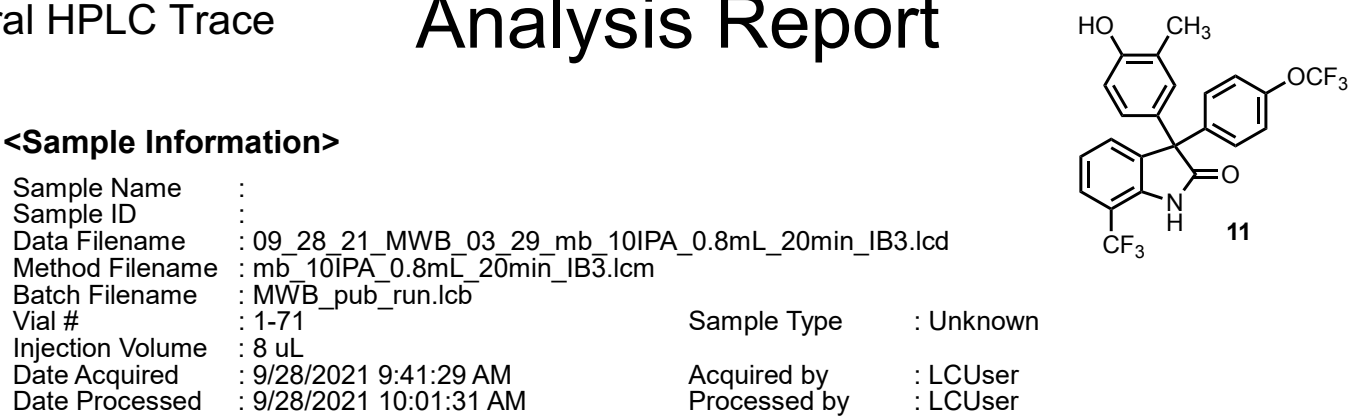

\section{$<$ Chromatogram >}

mAU

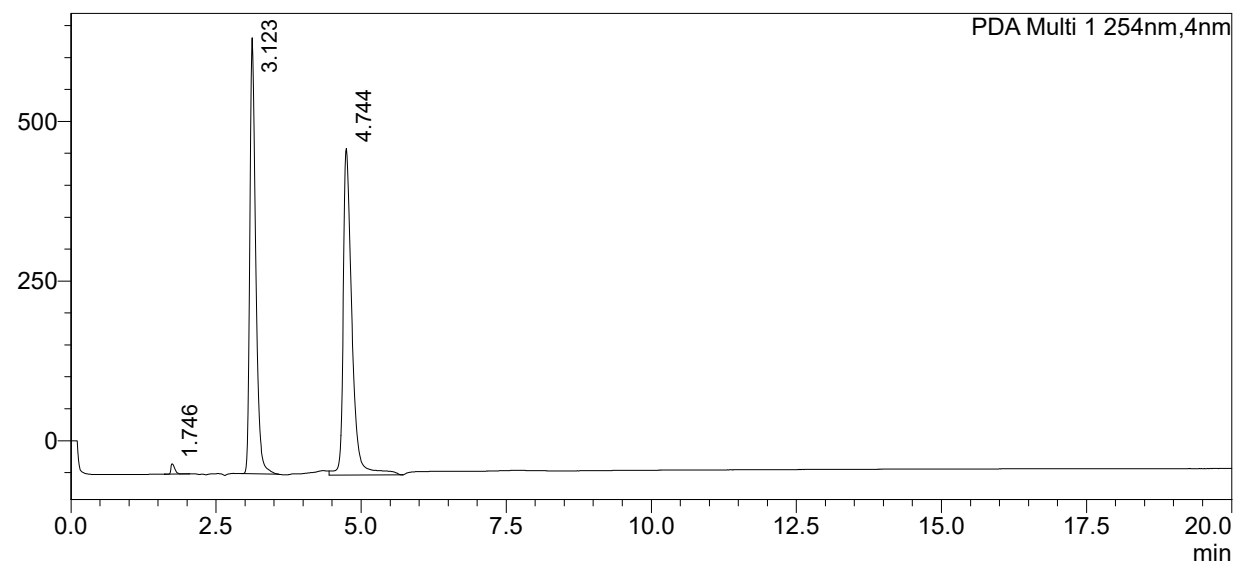

<Peak Table>

\begin{tabular}{|c|c|c|c|c|c|c|}
\hline \multicolumn{7}{|c|}{$1254 \mathrm{~nm}$} \\
\hline Peak\# & Ret. Time & Area & Height & Conc. & Area $\%$ & Height $\%$ \\
\hline 1 & 1.746 & 76247 & 16052 & 0.000 & 0.740 & 1.326 \\
\hline 2 & 3.123 & 4891103 & 682588 & 0.000 & 47.490 & 56.393 \\
\hline 3 & 4.744 & 5331875 & 511764 & 0.000 & 51.770 & 42.280 \\
\hline Total & & 10299224 & 1210404 & & 100.000 & 100.000 \\
\hline
\end{tabular}




\section{Chiral HPLC Trace}

\section{Analysis Report}

<Sample Information>

Sample Name

Sample ID

Data Filename : 12-4-20 MPM 0119 Purity 7.5IPA 0.8mL_15min cell IB3_10uL.Icd

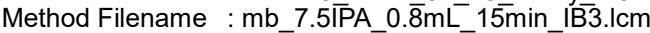

Batch Filename : Run this one.lcb

Vial \#

$1-40$

Sample Type

Unknown

: : $10 \mathrm{uL}$

Date Acquired : : 12/4/2020 12:38:17 PM

Date Processed $\quad$ : 12/4/2020 12:53:20 PM

Acquired by : : LCUser

Processed by : LCUser

\section{<Chromatogram >}

mAU

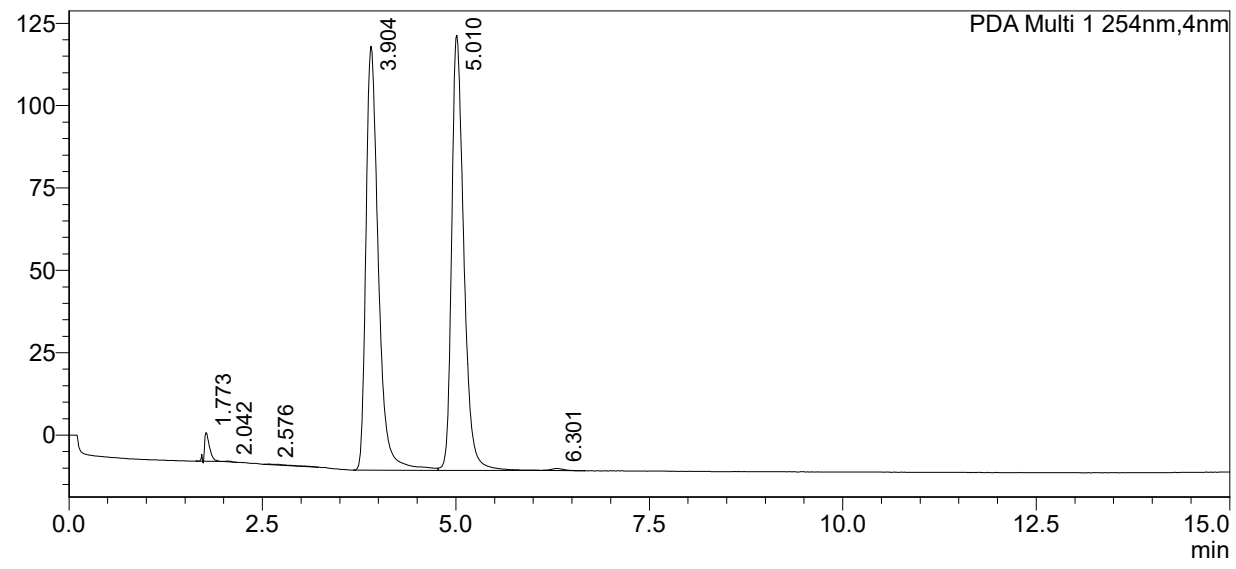

<Peak Table>

\begin{tabular}{|c|c|c|c|c|c|c|}
\hline \multicolumn{7}{|c|}{ PDA Ch1 254nm } \\
\hline & t. Time & Area & Height & Conc. & Area $\%$ & Height $\%$ \\
\hline 1 & 1.773 & 39244 & 8734 & 0.000 & 1.339 & 3.230 \\
\hline 2 & 2.042 & 1022 & 200 & 0.000 & 0.035 & 0.074 \\
\hline 3 & 2.576 & 4059 & 119 & 0.000 & 0.139 & 0.044 \\
\hline 4 & 3.904 & 1440300 & 128729 & 0.000 & 49.159 & 47.609 \\
\hline 5 & 5.010 & 1437995 & 132000 & 0.000 & 49.080 & 48.819 \\
\hline 6 & 6.301 & 7266 & 605 & 0.000 & 0.248 & 0.224 \\
\hline Total & & 2929885 & 270387 & & 100.000 & 100.000 \\
\hline
\end{tabular}




\section{Chiral HPLC Trace Analysis Report}

<Sample Information>

Sample Name

Sample ID

Data Filename : 12-4-20 MPM 0121 Purity 7.5IPA 0.8mL 15min cell IB3_10uL001.Icd

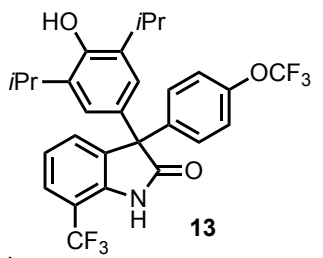

Method Filename : mb_7.5T्PA_0.8mL_15min_IB3.Icm

Batch Filename : Run this one.. $\mathrm{cb}$

Vial \# : : 1-41

Injection Volume :10

Date Acquired $\quad: 12 / 4 / 202012: 53: 51$ PM

Sample Type : Unknown

Date Processed $\quad: 12 / 4 / 20201: 08: 53$ PM

Acquired by : : LCUser

Processed by : : LCUser

\section{<Chromatogram>}

mAU

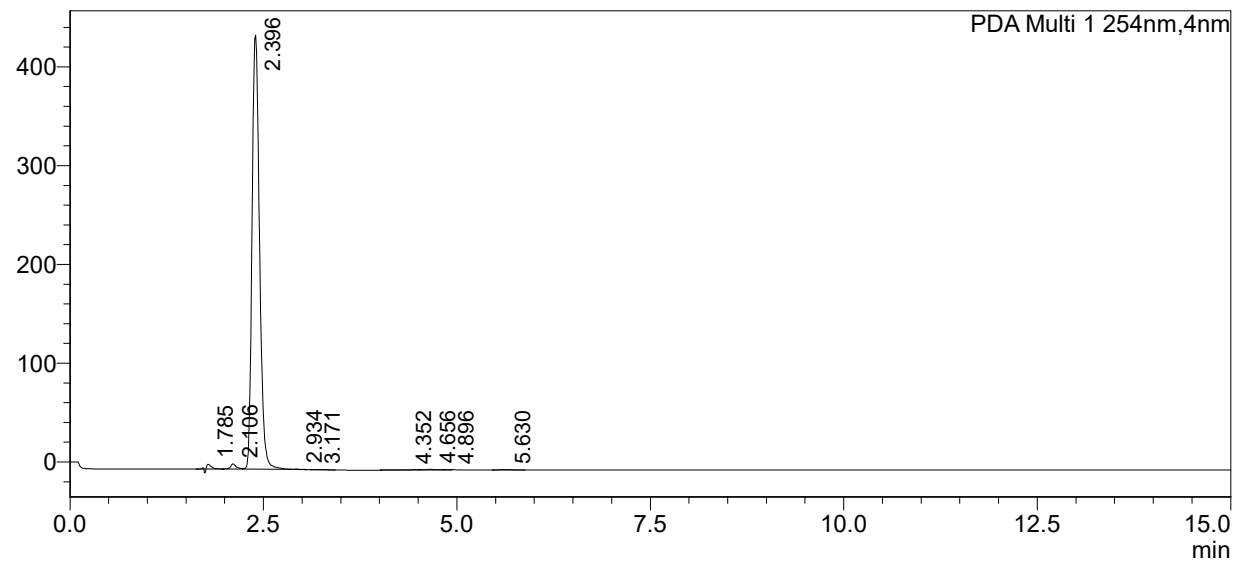

<Peak Table>

PDA Ch1 254nm

\begin{tabular}{|r|r|r|r|r|r|r|}
\hline Peak\# Ret. Time & \multicolumn{1}{|c|}{ Area } & Height & Conc. & Area\% & \multicolumn{1}{c|}{ Height\% } \\
\hline 1 & 1.785 & 17811 & 4853 & 0.000 & 0.588 & 1.076 \\
\hline 2 & 2.106 & 27261 & 5195 & 0.000 & 0.900 & 1.152 \\
\hline 3 & 2.396 & 2964563 & 439286 & 0.000 & 97.923 & 97.426 \\
\hline 4 & 2.934 & 1311 & 212 & 0.000 & 0.043 & 0.047 \\
\hline 5 & 3.171 & 1592 & 137 & 0.000 & 0.053 & 0.030 \\
\hline 6 & 4.352 & 4256 & 243 & 0.000 & 0.141 & 0.054 \\
\hline 7 & 4.656 & 7003 & 558 & 0.000 & 0.231 & 0.124 \\
\hline 8 & 4.896 & 1154 & 177 & 0.000 & 0.038 & 0.039 \\
\hline 9 & 5.630 & 2484 & 230 & 0.000 & 0.082 & 0.051 \\
\hline Total & & 3027434 & 450892 & & 100.000 & 100.000 \\
\hline
\end{tabular}




\section{Chiral HPLC Trace Analysis Report}

<Sample Information>

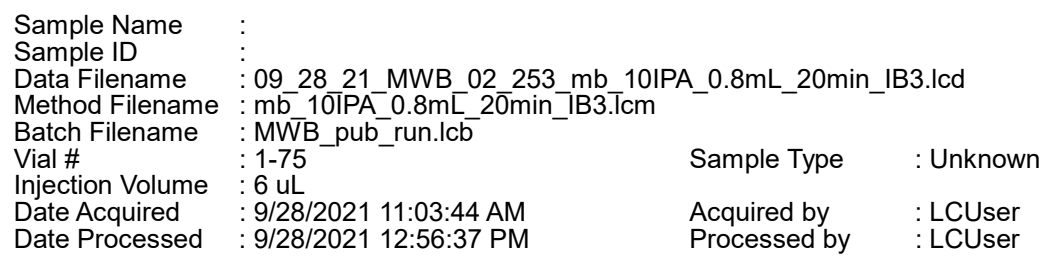

<Chromatogram>

mAU

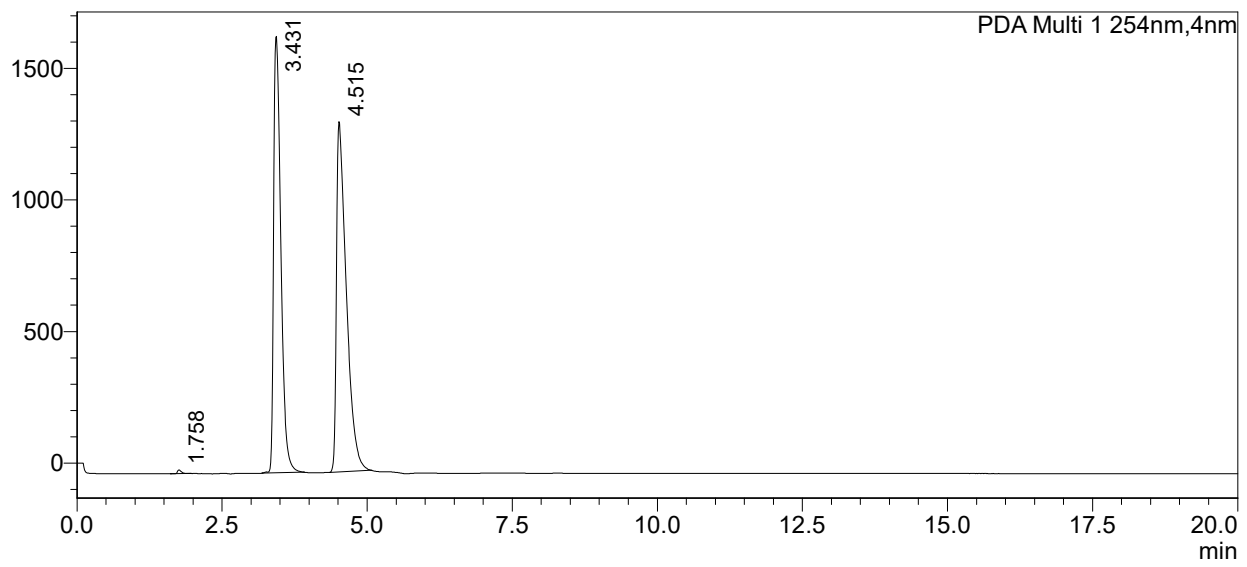

<Peak Table>

PDA Ch1 254nm

\begin{tabular}{|r|r|r|r|r|r|r|}
\hline Peak\# & Ret. Time & \multicolumn{1}{c|}{ Area } & Height & Conc. & Area\% & \multicolumn{1}{|c|}{ Height $\%$} \\
\hline 1 & 1.758 & 62177 & 13631 & 0.000 & 0.205 & 0.454 \\
\hline 2 & 3.431 & 14488904 & 1658493 & 0.000 & 47.811 & 55.220 \\
\hline 3 & 4.515 & 15753161 & 1331279 & 0.000 & 51.983 & 44.326 \\
\hline Total & & 30304242 & 3003403 & & 100.000 & 100.000 \\
\hline
\end{tabular}




\section{Analysis Report}

<Sample Information>
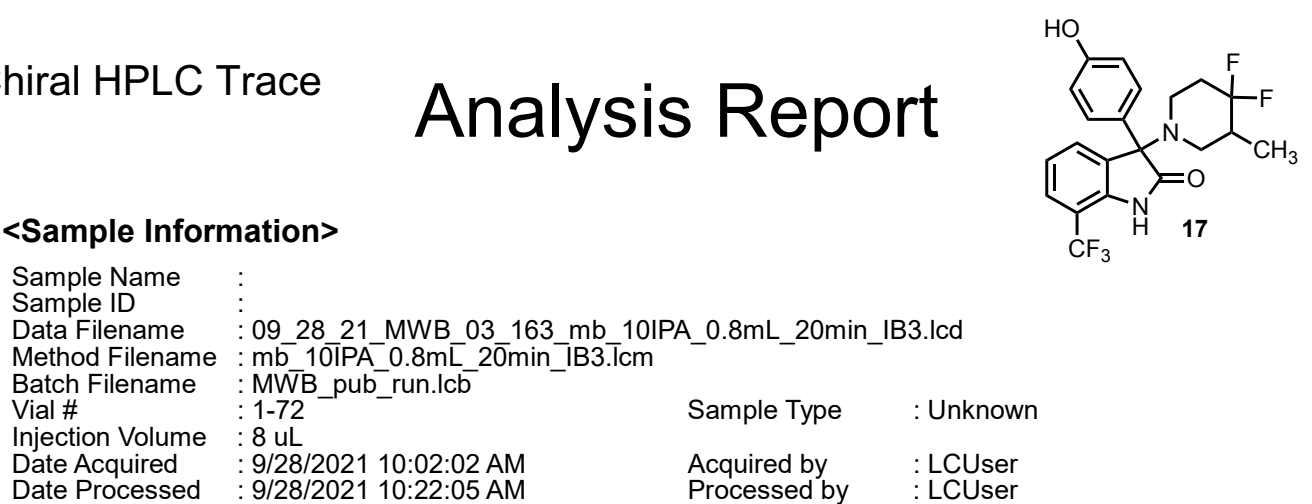

\section{$<$ Chromatogram >}

mAU

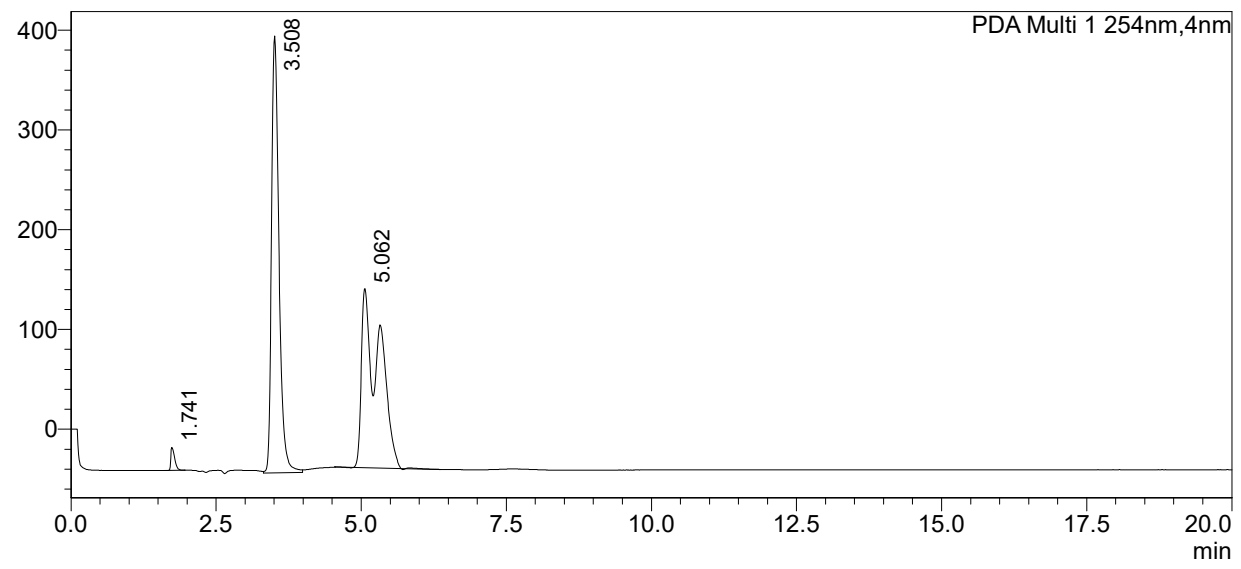

<Peak Table>

PDA Ch1 254nm

\begin{tabular}{|r|r|r|r|r|r|r|}
\hline Peak\# Ret. Time & \multicolumn{1}{|c|}{ Area } & \multicolumn{1}{c|}{ Height } & Conc. & \multicolumn{1}{|c|}{ Area\% } & Height\% \\
\hline 1 & 1.741 & 107163 & 22941 & 0.000 & 1.373 & 3.583 \\
\hline 2 & 3.508 & 3897845 & 437857 & 0.000 & 49.935 & 68.378 \\
\hline 3 & 5.062 & 3800821 & 179550 & 0.000 & 48.692 & 28.039 \\
\hline Total & & 7805830 & 640349 & & 100.000 & 100.000 \\
\hline
\end{tabular}




\section{Chiral HPLC Trace}

\section{Analysis Report}

<Sample Information>
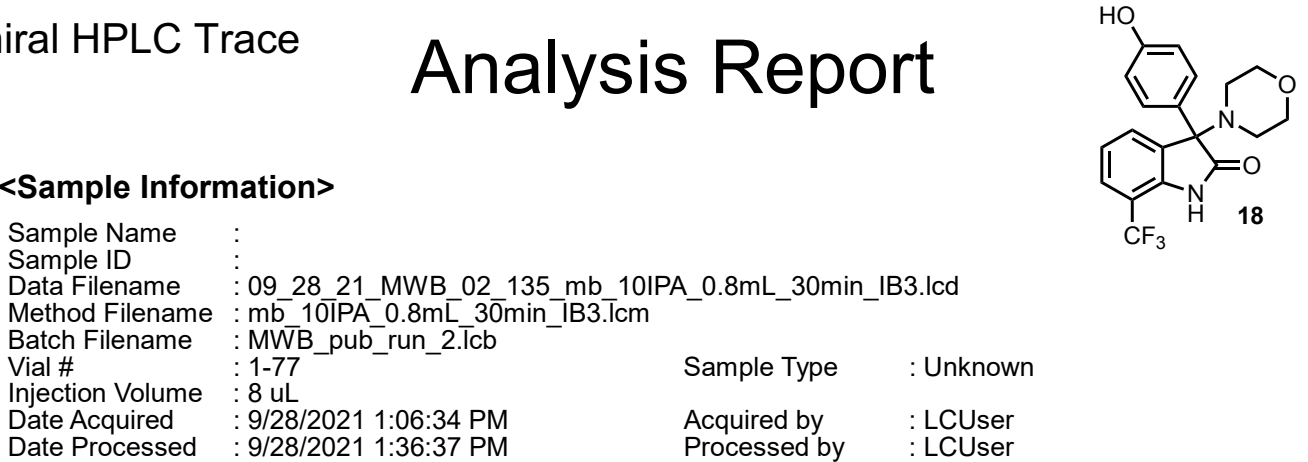

\section{<Chromatogram>}

mAU

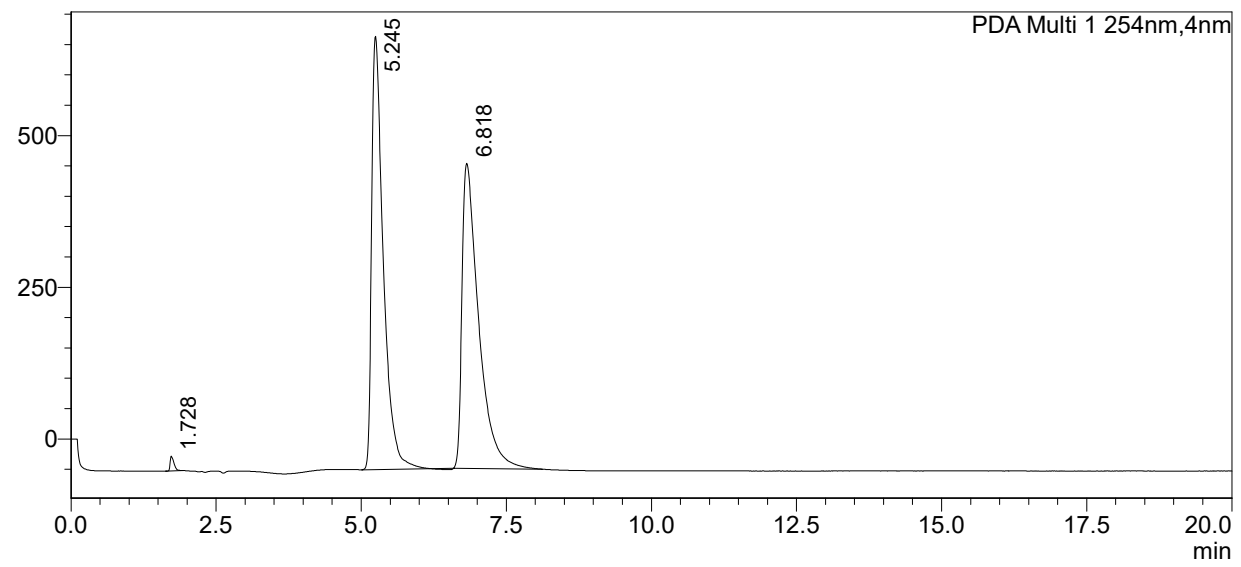

<Peak Table>

\begin{tabular}{|c|c|c|c|c|c|c|}
\hline \multicolumn{7}{|r|}{ Height\% } \\
\hline 1 & 1.728 & 112070 & 24119 & 0.000 & 0.558 & 1.944 \\
\hline 2 & 5.245 & 9949383 & 713751 & 0.000 & 49.502 & 57.532 \\
\hline 3 & 6.818 & 10037689 & 502736 & 0.000 & 49.941 & 40.523 \\
\hline Total & & 20099142 & 1240606 & & 100.000 & 100.000 \\
\hline
\end{tabular}




\section{Chiral HPLC Trace Analysis Report}

<Sample Information>
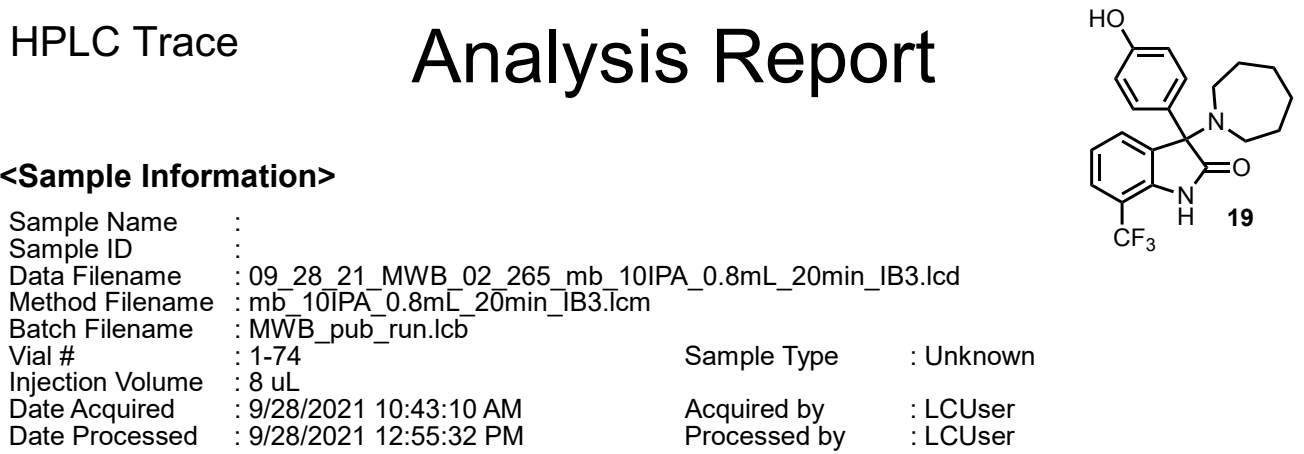

\section{$<$ Chromatogram $>$}

mAU

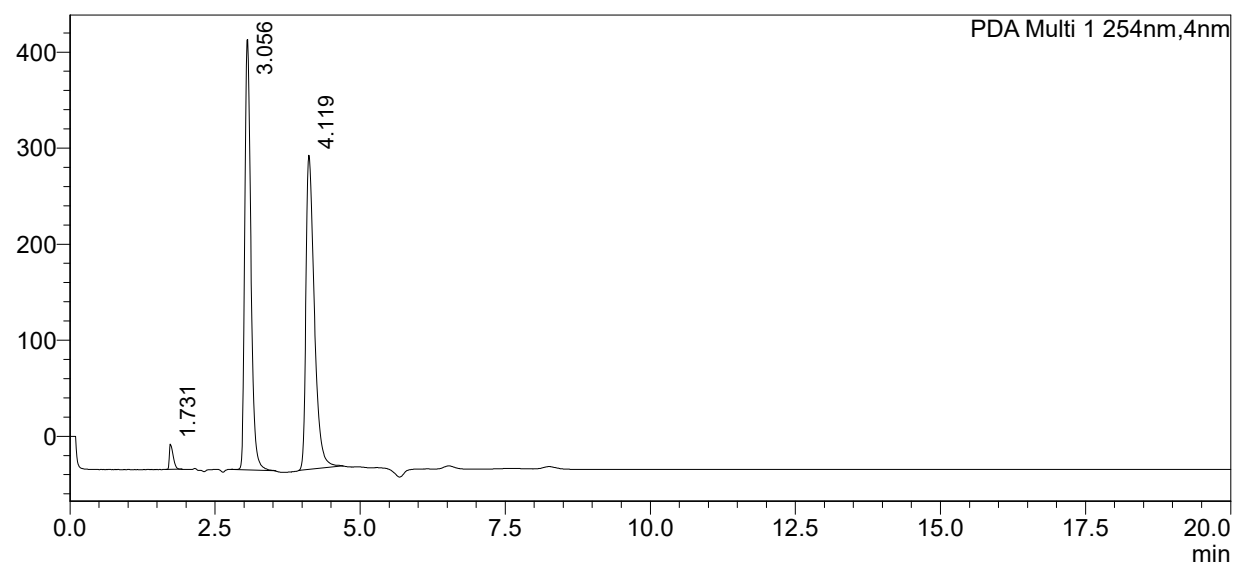

<Peak Table>

PDA Ch1 254nm

Peak\# Ret. Time

\begin{tabular}{|c|c|c|c|c|c|c|}
\hline eak\# & Ret. Time & Area & Height & Conc. & Area\% & Height $\%$ \\
\hline 1 & 1.731 & 124262 & 26374 & 0.000 & 1.860 & 3.290 \\
\hline 2 & 3.056 & 3278062 & 448040 & 0.000 & 49.056 & 55.889 \\
\hline 3 & 4.119 & 3279972 & 327253 & 0.000 & 49.085 & 40.822 \\
\hline Total & & 6682296 & 801668 & & 100.000 & 10 \\
\hline
\end{tabular}




\section{Chiral HPLC Trace Analysis Report}

\section{<Sample Information>}

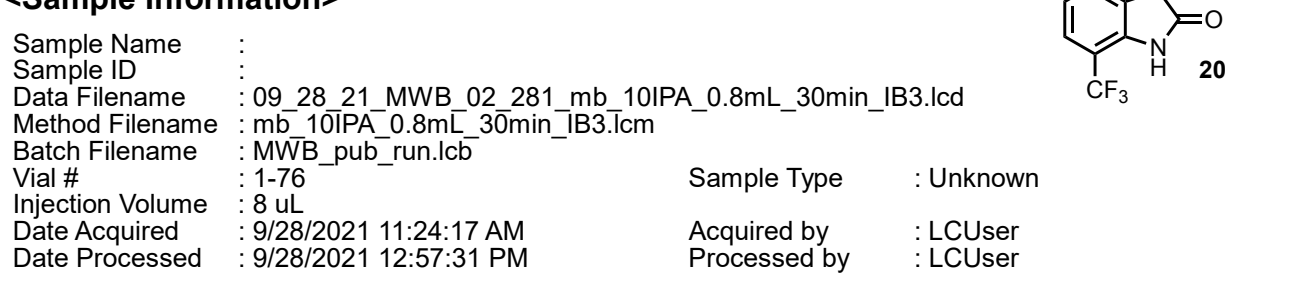

\section{$<$ Chromatogram $>$}

mAU

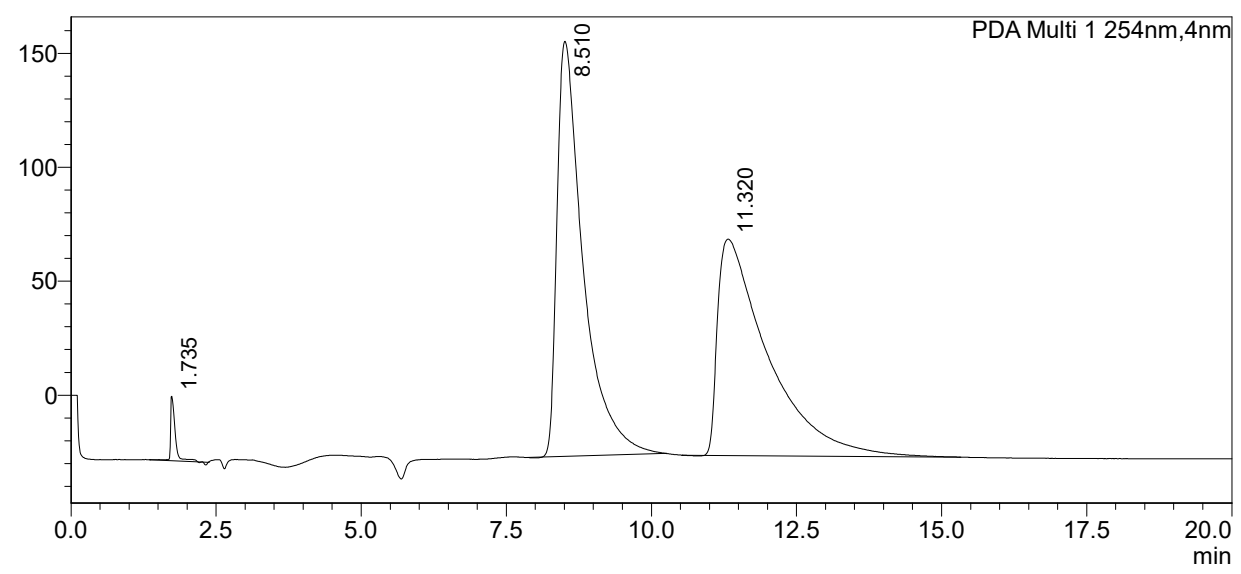

<Peak Table>

PDA Ch1 254nm

\begin{tabular}{|r|r|r|r|r|r|r|}
\hline Peak\# Ret. Time & \multicolumn{1}{|c|}{ Area } & \multicolumn{1}{c|}{ Height } & Conc. & \multicolumn{1}{|c|}{ Area $\%$} & \multicolumn{1}{|c|}{ Height $\%$} \\
\hline 1 & 1.735 & 154255 & 28300 & 0.000 & 1.331 & 9.269 \\
\hline 2 & 8.510 & 5705171 & 182101 & 0.000 & 49.229 & 59.641 \\
\hline 3 & 11.320 & 5729535 & 94929 & 0.000 & 49.440 & 31.091 \\
\hline Total & & 11588960 & 305331 & & 100.000 & 100.000 \\
\hline
\end{tabular}




\section{Chiral HPLC Trace Analysis Report}

<Sample Information>

Sample Name

Sample ID

Data Filename : : 092821 MWB $0307 \mathrm{mb}$ 10IPA 0.8mL 20min IB3.Icd

Method Filename : mb_10IPA_0.8m[_20̄min_IB 3 .Icm

Batch Filename : MWBB_pub_run.lcb

Vial \# $: 1-73$

$\begin{array}{lll}\text { Date Acquired } & : 9 / 28 / 2021 & 10: 22: 36 ~ A M \\ \text { Date Processed } & : 9 / 28 / 2021 & 12: 54: 43 \text { PM }\end{array}$

$\begin{array}{ll}\text { Sample Type } & \text { : Unknown } \\ \text { Acquired by } & : \text { LCUser } \\ \text { Processed by } & \text { : LCUser }\end{array}$

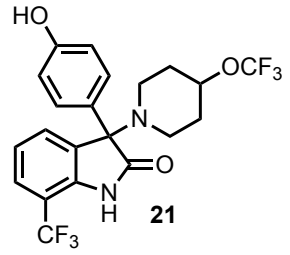

<Chromatogram>

mAU

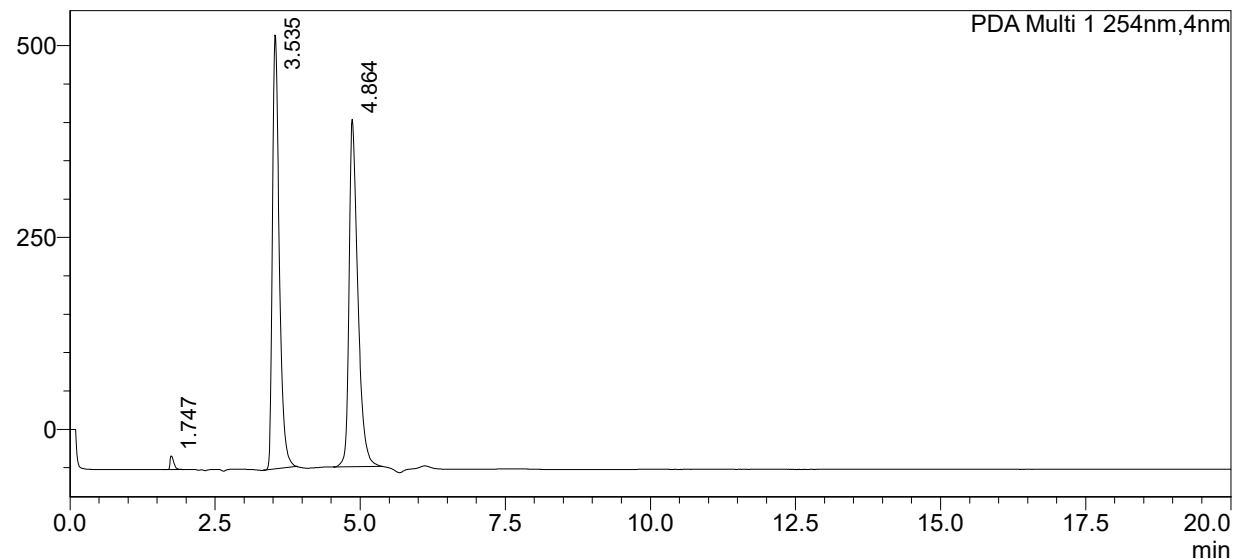

<Peak Table>

PDA Ch1 254nm

\begin{tabular}{|r|r|r|r|r|r|r|}
\hline Peak\# & Ret. Time & \multicolumn{1}{|c|}{ Area } & Height & Conc. & Area\% & \multicolumn{1}{|c|}{ Height $\%$} \\
\hline 1 & 1.747 & 79898 & 17749 & 0.000 & 0.842 & 1.713 \\
\hline 2 & 3.535 & 4632723 & 565552 & 0.000 & 48.831 & 54.582 \\
\hline 3 & 4.864 & 4774625 & 452846 & 0.000 & 50.327 & 43.705 \\
\hline Total & & 9487246 & 1036147 & & 100.000 & 100.000 \\
\hline
\end{tabular}




\section{Chiral HPLC Trace Analysis Report}

\section{<Sample Information>}

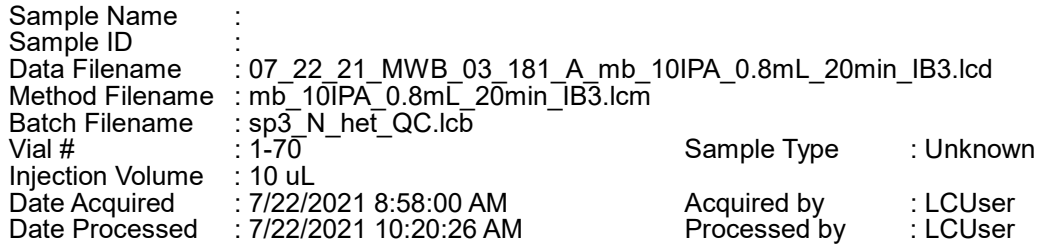

\section{$<$ Chromatogram $>$}

$\mathrm{mAU}$

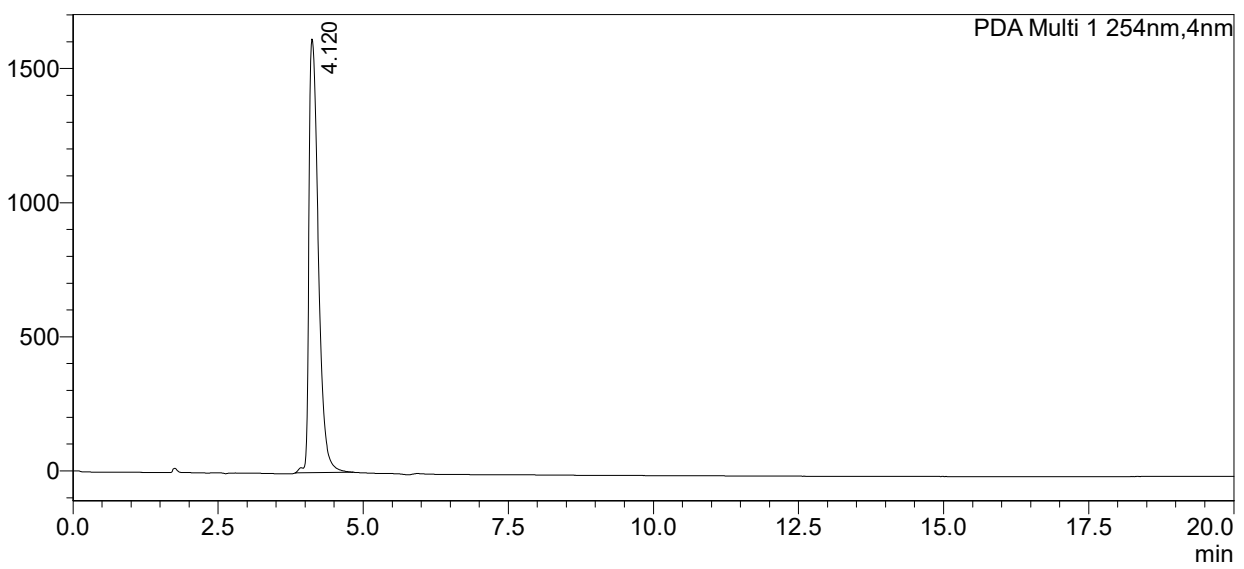

<Peak Table>

PDA Ch1 254nm

\begin{tabular}{|r|r|r|r|r|r|r|}
\hline Peak\# Ret. Time & \multicolumn{1}{|c|}{ Area } & Height & \multicolumn{1}{|c|}{ Conc. } & \multicolumn{1}{|c|}{ Area\% } & \multicolumn{1}{|c|}{ Height\% } \\
\hline 1 & 4.120 & 18191621 & 1616722 & 0.000 & 100.000 & 100.000 \\
\hline Total & & 18191621 & 1616722 & & 100.000 & 100.000 \\
\hline
\end{tabular}




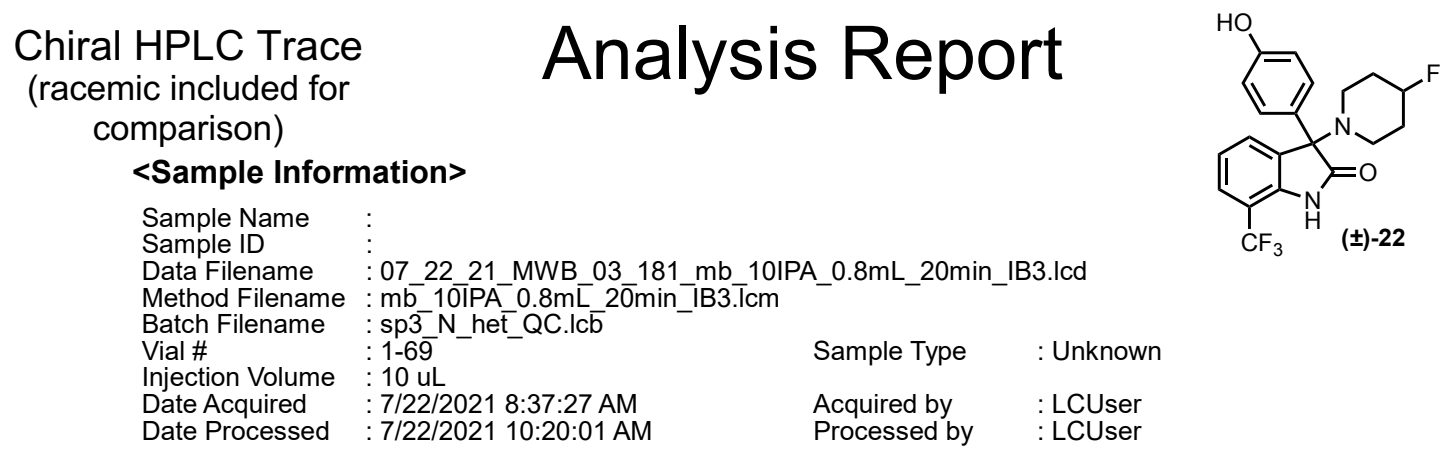

\section{<Chromatogram>}

mAU

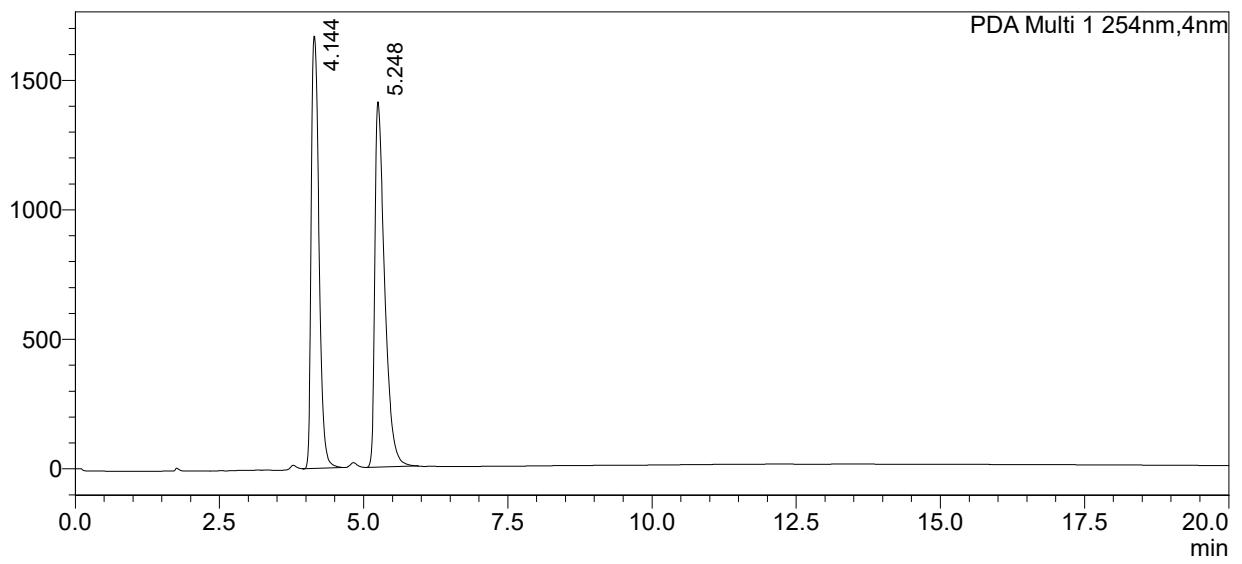

<Peak Table>

PDA Ch1 254nm

Peak\# Ret. Time

\begin{tabular}{|r|r|r|r|r|r|r|}
\hline Peak\# Ret. Time & \multicolumn{1}{|c|}{ Area } & Height & \multicolumn{1}{|c|}{ Conc. } & \multicolumn{1}{|c|}{ Area\% } & \multicolumn{1}{|c|}{ Height\% } \\
\hline 1 & 4.144 & 15644643 & 1669167 & 0.000 & 48.441 & 54.196 \\
\hline 2 & 5.248 & 16651778 & 1410723 & 0.000 & 51.559 & 45.804 \\
\hline Total & & 32296421 & 3079889 & & 100.000 & 100.000 \\
\hline
\end{tabular}




\section{Chiral HPLC Trace Analysis Report}

\section{$<$ Sample Information>}
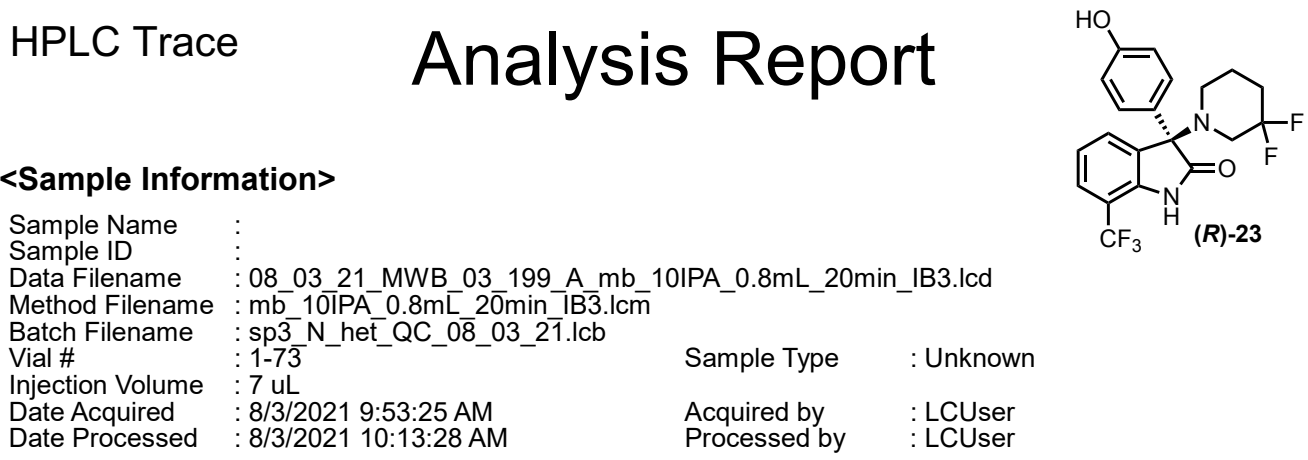

\section{$<$ Chromatogram>}

$\mathrm{mAU}$

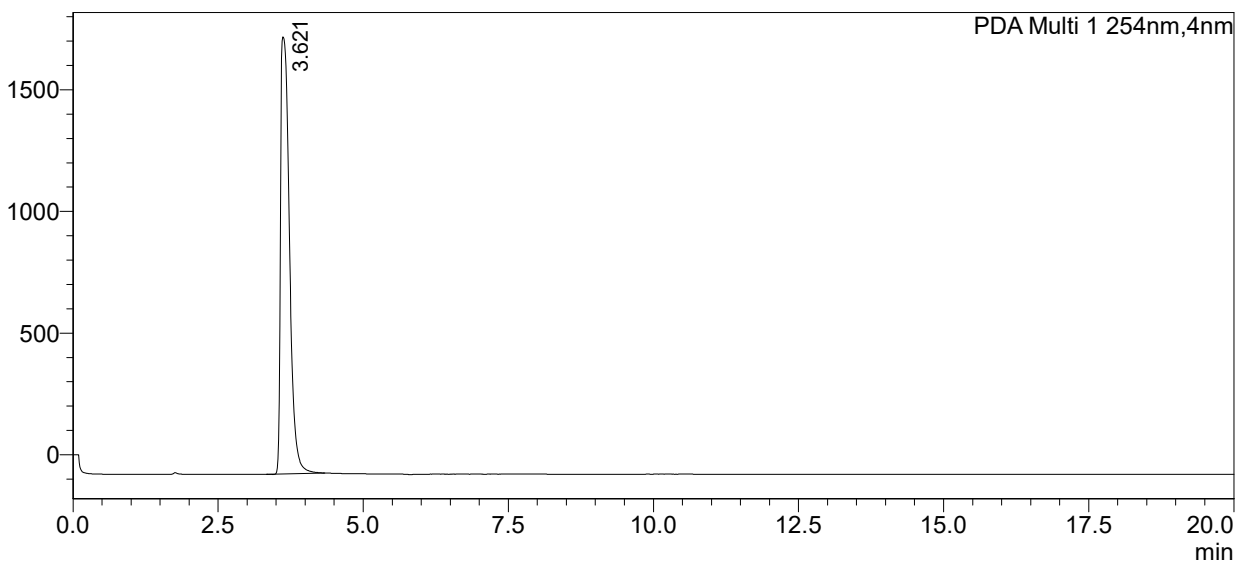

<Peak Table>

PDA Ch1 254nm

\begin{tabular}{|r|r|r|r|r|r|r|}
\hline Peak\# & Ret. Time & \multicolumn{1}{|c|}{ Area } & Height & \multicolumn{1}{|c|}{ Conc. } & \multicolumn{1}{|c|}{ Area\% } & \multicolumn{1}{|c|}{ Height $\%$} \\
\hline 1 & 3.621 & 19264037 & 1795792 & 0.000 & 100.000 & 100.000 \\
\hline Total & & 19264037 & 1795792 & & 100.000 & 100.000 \\
\hline
\end{tabular}




\section{Chiral HPLC Trace \\ (racemic included for \\ Analysis Report}

comparison)

\section{$<$ Sample Information>}

Sample Name

Sample ID

Data Filename

:08_03 21_MWB_03_199_mb_10IPA_0.8mL_20min_IB3.Icd

Method Filename : mb_10IPA_0.8mL_20̄min_IB3.Tcm

Batch Filename : sp3 N het QC_08_03 21.lcb

Vial

$1-7 \overline{2}$

Injection Volume $: 7 \mathrm{uL}$

Date Acquired : : 8/3/2021 9:32:52 AM

Date Processed $\quad: 8 / 3 / 2021$ 11:56:43 AM

Sample Type : Unknown

Acquired by : : LCUser

Processed by : : LCUser

\section{$<$ Chromatogram $>$}

$\mathrm{mAU}$

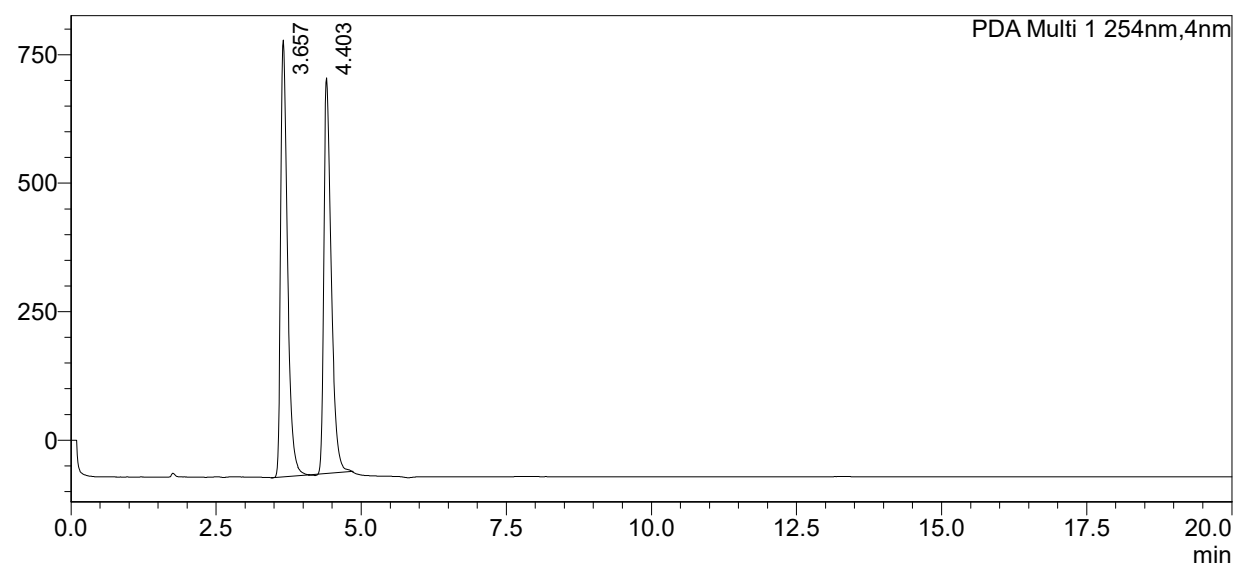

<Peak Table>

PDA Ch1 254nm

\begin{tabular}{|r|r|r|r|r|r|r|}
\hline Peak\# & Ret. Time & \multicolumn{1}{|c|}{ Area } & \multicolumn{1}{|c|}{ Height } & Conc. & \multicolumn{1}{c|}{ Area\% } & \multicolumn{1}{c|}{ Height $\%$} \\
\hline 1 & 3.657 & 6957675 & 849571 & 0.000 & 50.443 & 52.469 \\
\hline 2 & 4.403 & 6835395 & 769608 & 0.000 & 49.557 & 47.531 \\
\hline Total & & 13793070 & 1619179 & & 100.000 & 100.000 \\
\hline
\end{tabular}




\section{Chiral HPLC Trace Analysis Report}

<Sample Information>
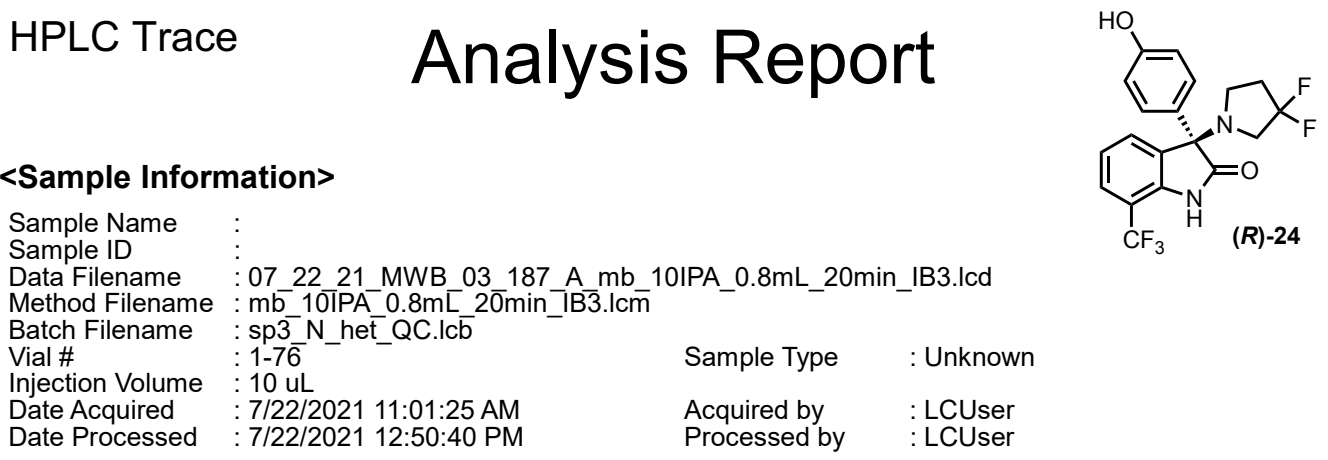

\section{$<$ Chromatogram>}

mAU

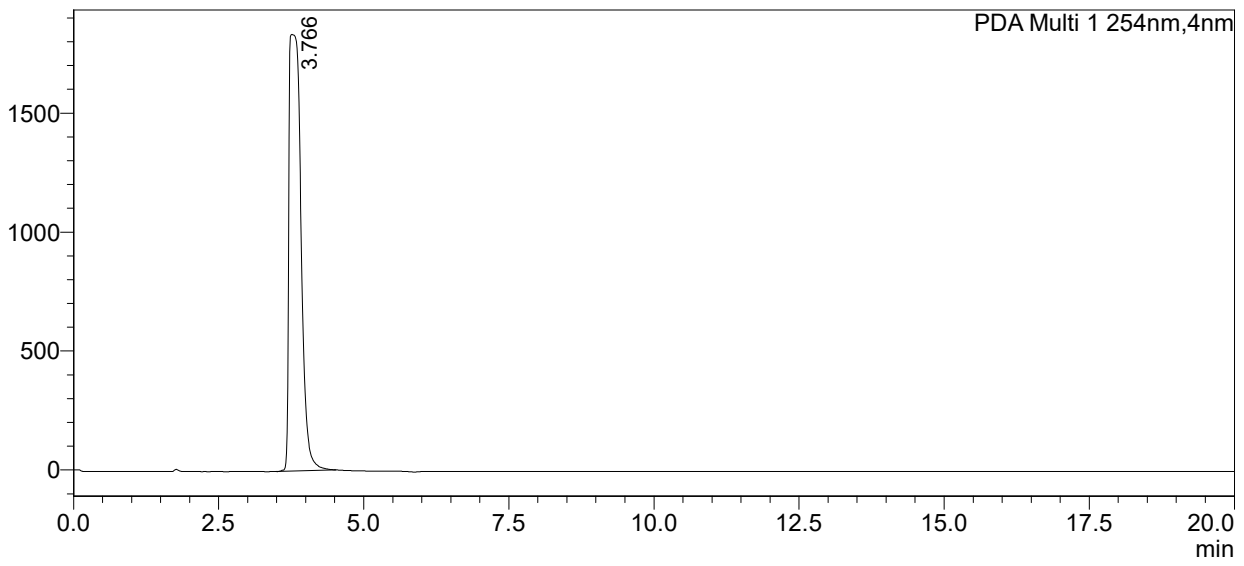

<Peak Table>

PDA Ch1 254nm

\begin{tabular}{|c|c|c|c|c|c|c|}
\hline Peak\# & t. Time & Area & Height & Conc. & Area\% & Height $\%$ \\
\hline 1 & 3.766 & 26499898 & 1835371 & 0.000 & 100.000 & 100.000 \\
\hline Total & & 26499898 & 1835371 & & 100.000 & 100.000 \\
\hline
\end{tabular}




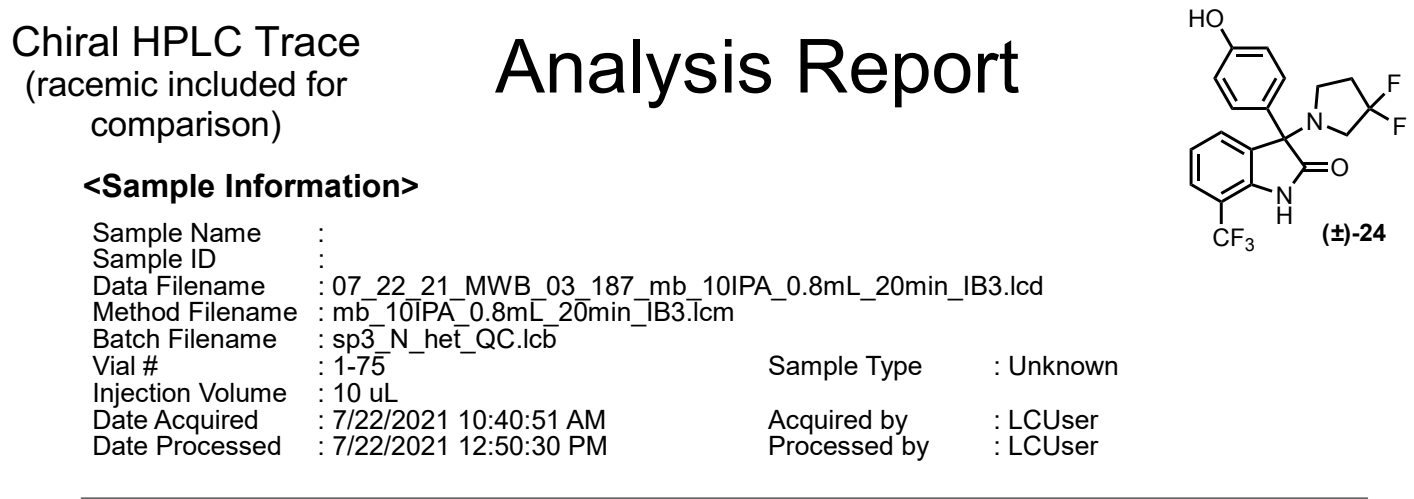

\section{<Chromatogram>}

mAU

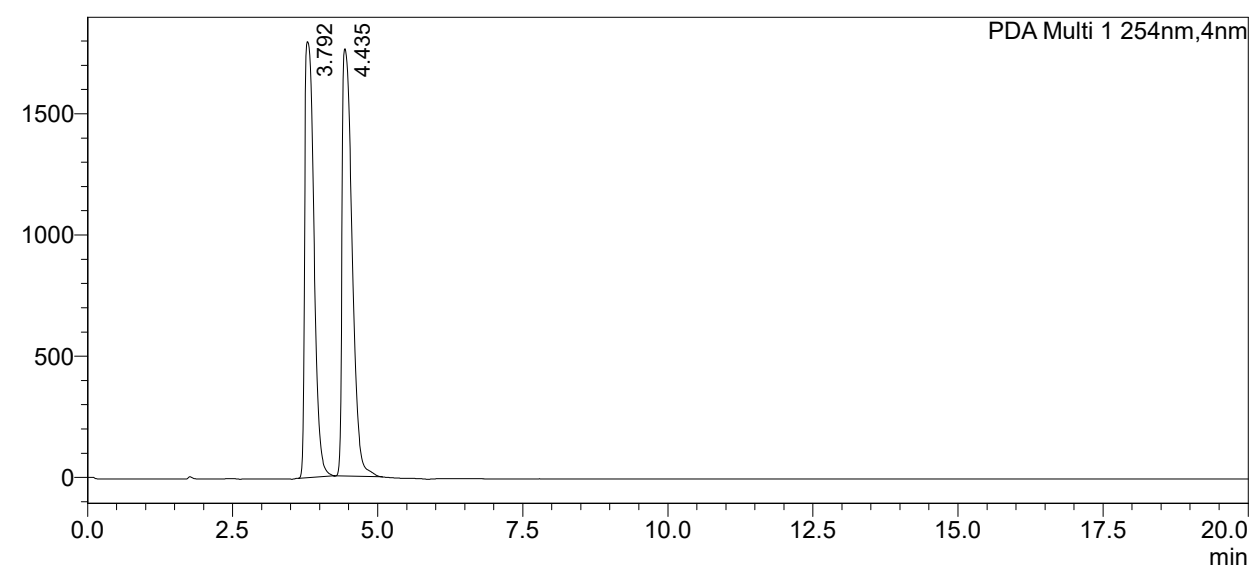

<Peak Table>

PDA Ch1 254nm

\begin{tabular}{|r|r|r|r|r|r|r|}
\multicolumn{2}{|c|}{ PDA Cak\# Ret. Time } & \multicolumn{1}{c|}{ Area } & Height & Conc. & \multicolumn{1}{c|}{ Area\% } & \multicolumn{1}{|c|}{ Height $\%$} \\
\hline 1 & 3.792 & 19882139 & 1797554 & 0.000 & 48.358 & 50.499 \\
\hline 2 & 4.435 & 21232726 & 1762048 & 0.000 & 51.642 & 49.501 \\
\hline Total & & 41114865 & 3559601 & & 100.000 & 100.000 \\
\hline
\end{tabular}




\section{Chiral HPLC Trace Analysis Report}

<Sample Information>

Sample Name

Sample ID

Data Filename :08_03_21_MWB_03_195_A_mb_10IPA_0.8mL_20min_IB3.Icd

Method Filename : $\mathrm{mb}$ 10IPA $0.8 \mathrm{~mL} 20 \overline{\mathrm{min}}-\mathrm{IB} \overline{\mathrm{B}} . \mathrm{Icm}$

Batch Filename : sp3_N_het_QC_08_03_21.Icb

$\begin{array}{lll}\text { Vial \# } & : 1-70 \\ \text { Injection Volume } & : 7 \text { uL }\end{array}$

Date Acquired $\quad: 8 / 3 / 20218: 51: 47 \mathrm{AM}$

Sample Type : Unknown

Date Processed $\quad: 8 / 3 / 2021$ 9:11:49 AM

Acquired by : : LCUser

Processed by : LCUser

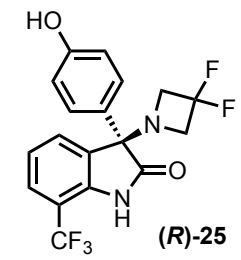

\section{<Chromatogram>}

mAU

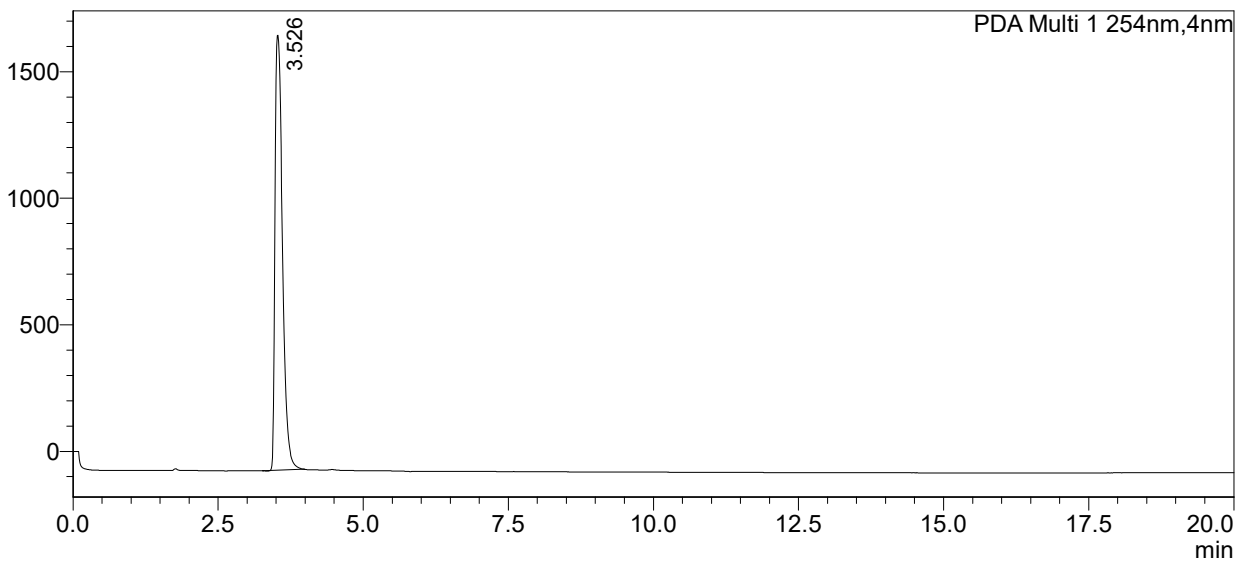

<Peak Table>

PDA Ch1 254nm

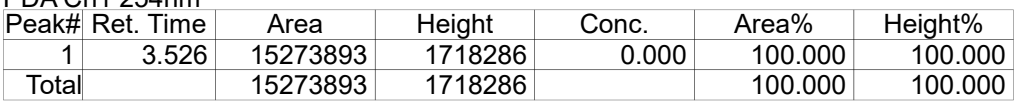




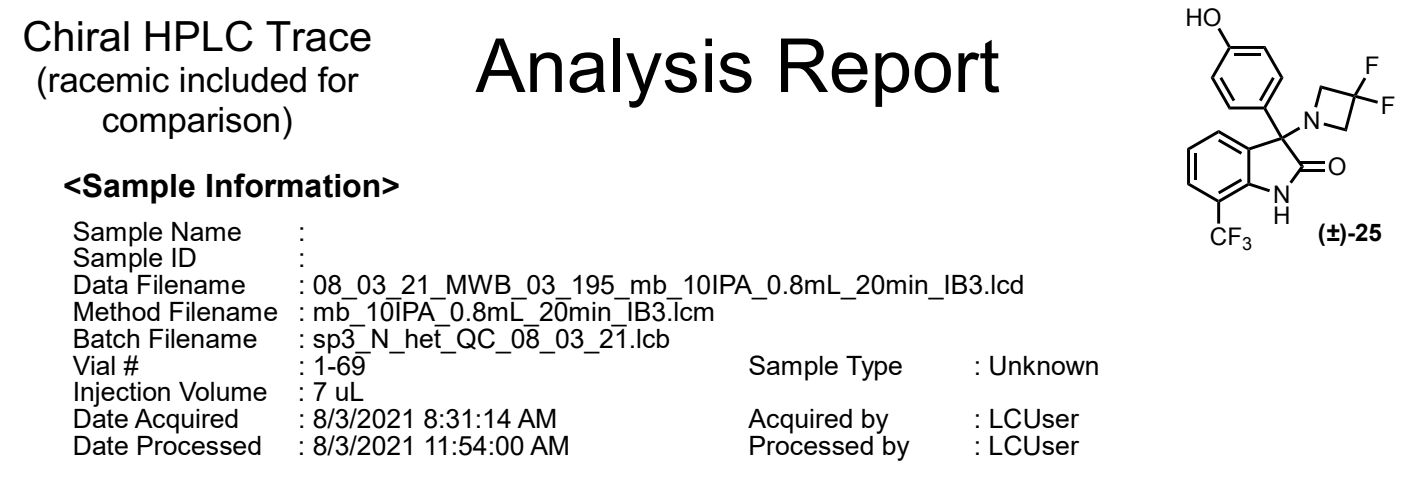

<Chromatogram>

mAU

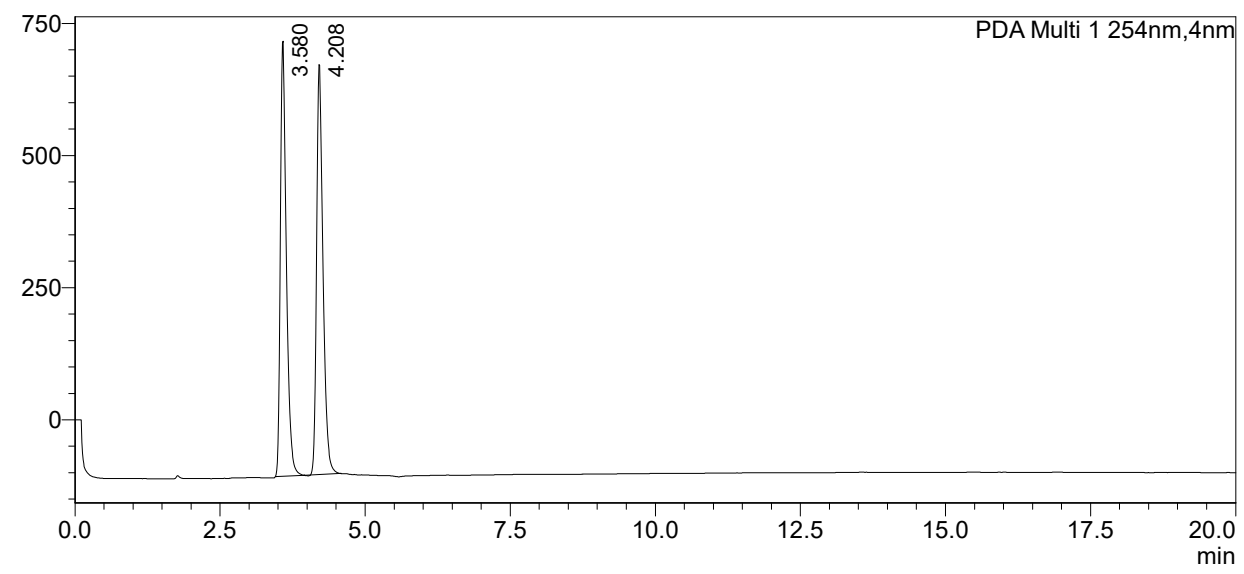

<Peak Table>

\begin{tabular}{|c|c|c|c|c|c|c|}
\hline \multicolumn{2}{|c|}{$\begin{array}{l}\text { PDA Ch1 } 254 \mathrm{~nm} \\
\text { Peak\# Ret. Time }\end{array}$} & Area & Height & Conc & Area\% & Height\% \\
\hline 1 & 3.580 & 5974262 & 822845 & 0.000 & 49.928 & 51.481 \\
\hline 2 & 4.208 & 5991589 & 775509 & 0.000 & 50.072 & 48.519 \\
\hline Total & & 11965851 & 1598354 & & 100.000 & 100.000 \\
\hline
\end{tabular}




\section{Chiral HPLC Trace}

\section{Analysis Report}

<Sample Information>
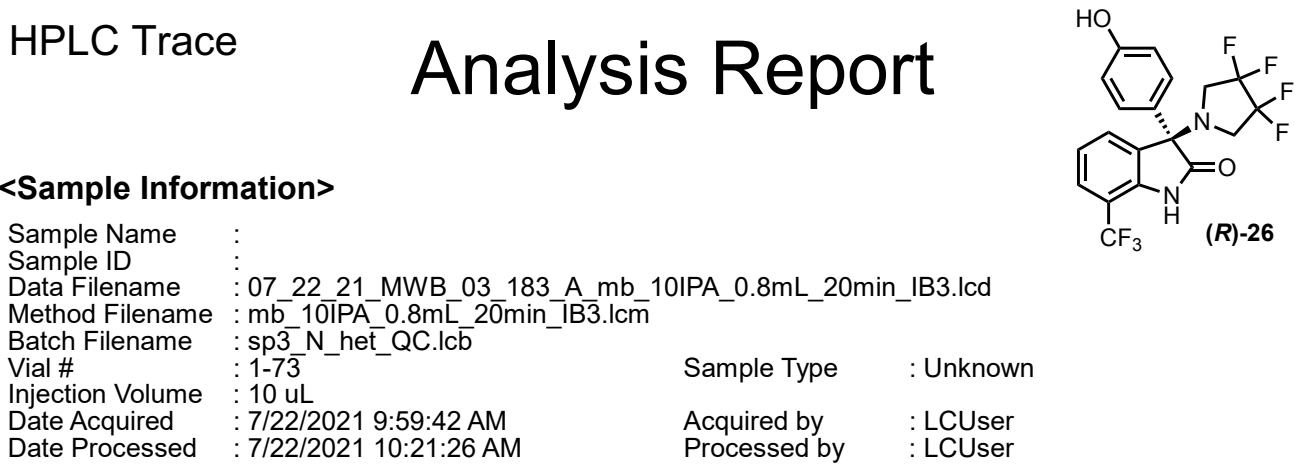

\section{$<$ Chromatogram>}

$\mathrm{mAU}$

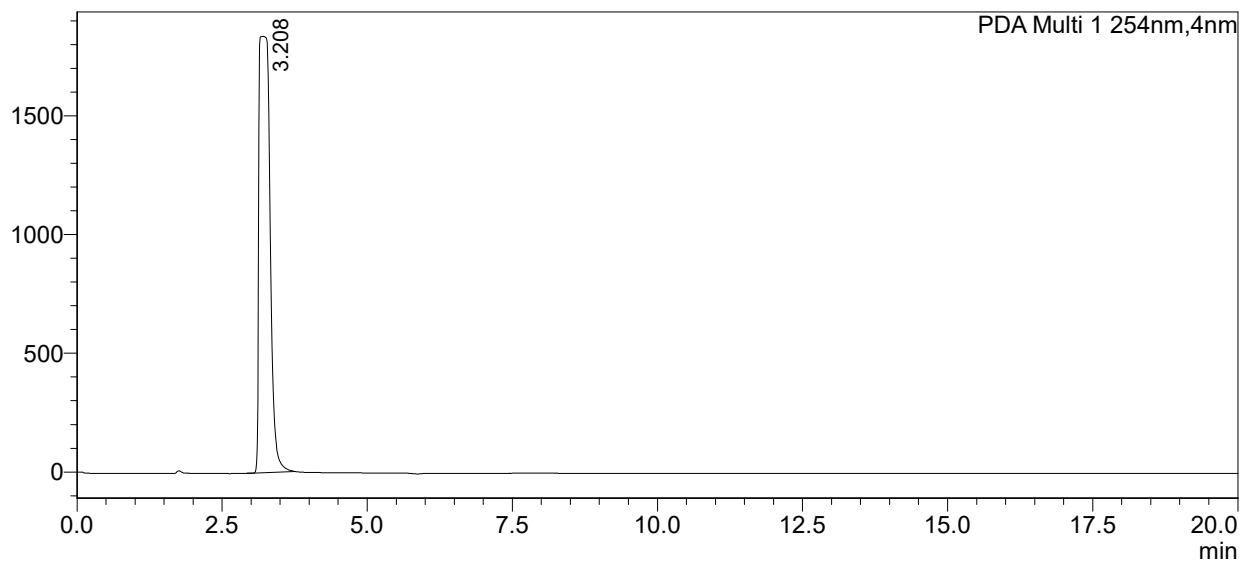

<Peak Table>

\begin{tabular}{|c|c|c|c|c|c|c|}
\hline \multicolumn{7}{|c|}{ PDA Ch1 } \\
\hline Peak\# & Ret. Time & Area & Height & Conc. & Area\% & Heig \\
\hline 1 & 3.208 & 24426537 & 1836814 & 0.000 & 100.000 & 000 \\
\hline Tota & & 24426537 & 1836814 & & 100.000 & 100.000 \\
\hline
\end{tabular}




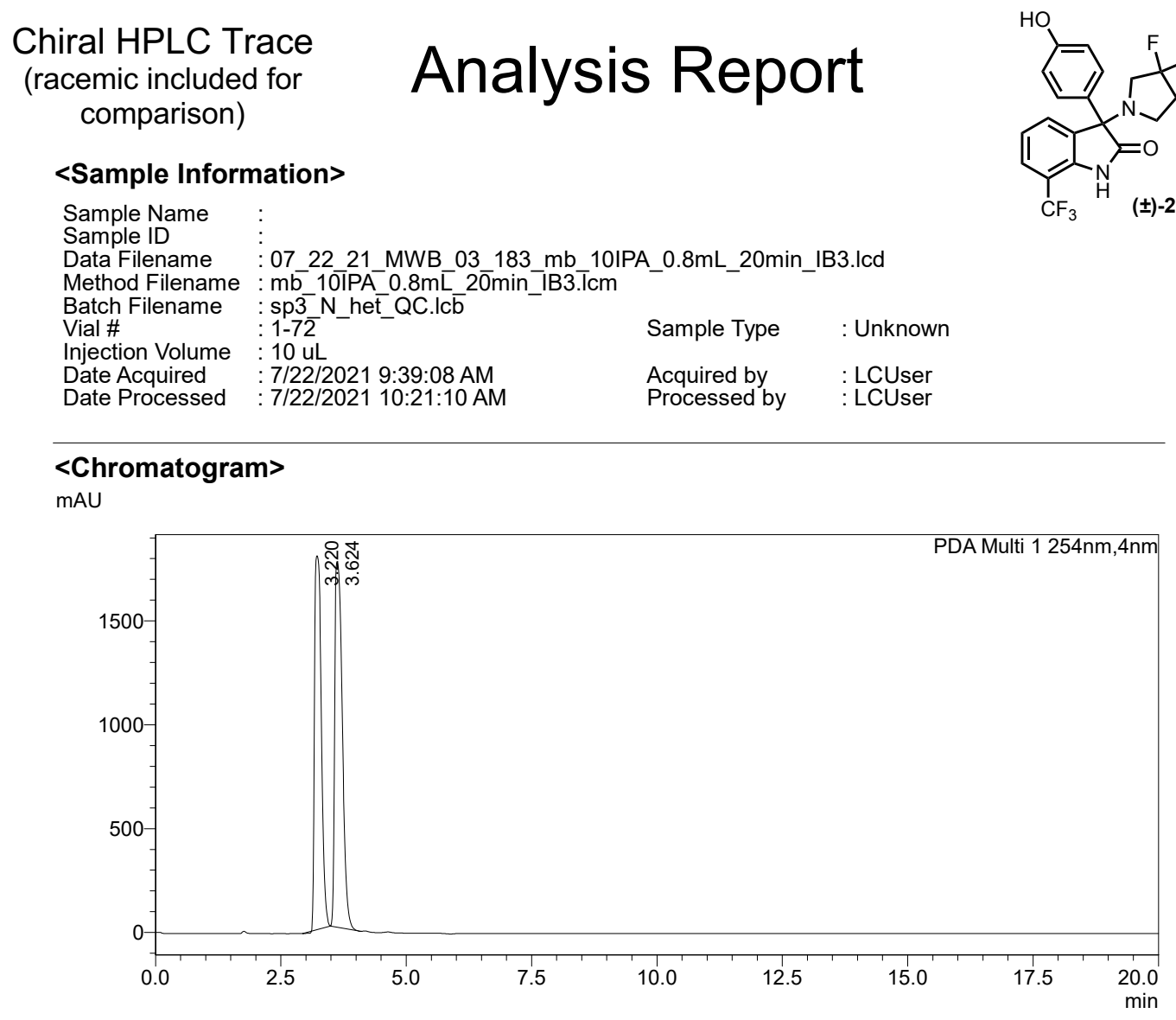

<Peak Table>

\begin{tabular}{|c|c|c|c|c|c|c|}
\hline \\
\hline & \multirow{3}{*}{$\begin{array}{c}\text { Area } \\
16857747 \\
18563698\end{array}$} & \multirow{3}{*}{$\begin{array}{l}\text { Height } \\
1799582 \\
1757997\end{array}$} & Conc. & Area $\%$ & Height $\%$ \\
\hline \multicolumn{2}{|c|}{$\begin{array}{rr}\text { Peak\# } & \text { Ret. Time } \\
1 & 3220\end{array}$} & & & \multirow{2}{*}{$\begin{array}{l}0.000 \\
0.000\end{array}$} & \multirow{2}{*}{$\begin{array}{l}47.592 \\
52.408\end{array}$} & \multirow{2}{*}{$\begin{array}{l}50.584 \\
49.416\end{array}$} \\
\hline \multirow{2}{*}{\multicolumn{2}{|c|}{\begin{tabular}{|r|r|}
2 & 3.624 \\
\end{tabular}}} & & & & & \\
\hline & & 35421445 & 3557579 & & 100.000 & 100.000 \\
\hline
\end{tabular}




\section{Chiral HPLC Trace Analysis Report}

<Sample Information>

Sample Name

Sample ID :

Data Filename $\quad: 07 \quad 22 \quad 21$ MWB $03 \quad 183$ B 2uL mb 10IPA 0.8mL_20min_IB3.Icd

Method Filename : $\mathrm{mb}$ 10IPA $0.8 \mathrm{~mL} 20 \mathrm{~min}{ }^{\mathrm{IB}} 3 . \mathrm{Icm}$

Batch Filename : $\mathrm{sp} 3 \mathrm{~N}$ het $\mathrm{QC}$ small.Icb

Vial \# $\quad: 1-7 \overline{4}$

Injection Volume $: 1 \mathrm{uL}$

Date Acquired : : 7/22/2021 12:54:09 PM

Date Processed $\quad:$ 7/22/2021 2:11:05 PM

$\begin{array}{ll}\text { Sample Type } & \text { : Unknown } \\ \text { Acquired by } & : \text { LCUser } \\ \text { Processed by } & : \text { LCUser }\end{array}$

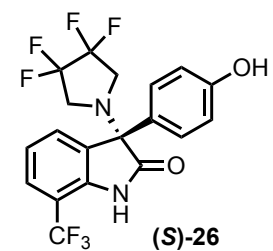

(S)-26

\section{<Chromatogram>}

mAU

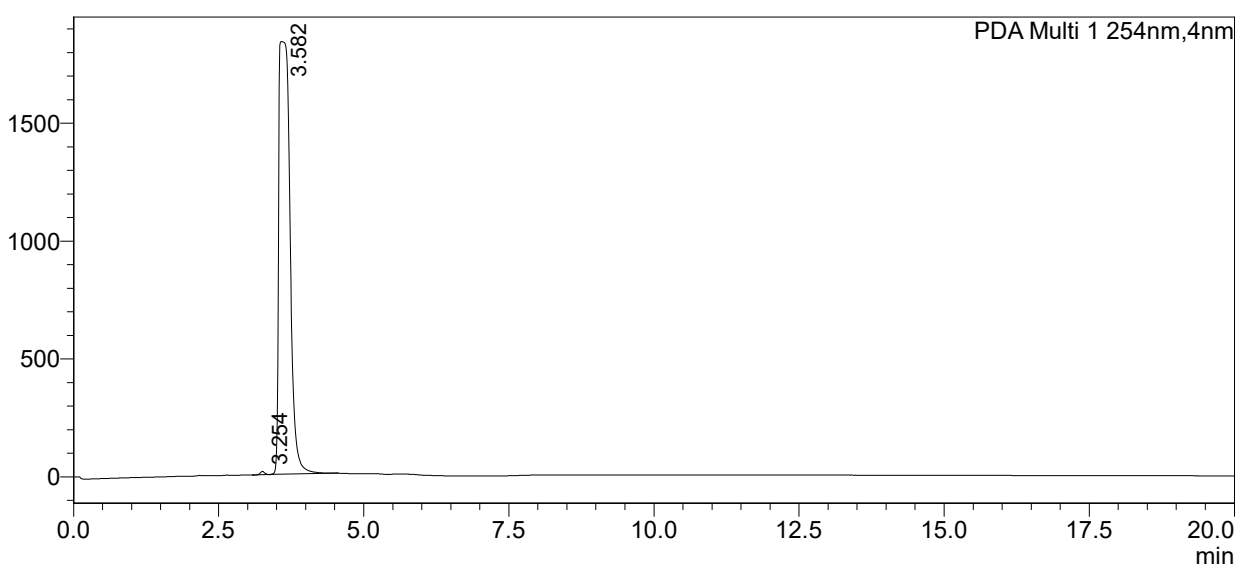

<Peak Table>

PDA Ch1 254nm

Peak\# Ret. Time

\begin{tabular}{|r|r|r|r|r|r|r|}
\hline Peak\# Ret. Time & \multicolumn{1}{|c|}{ Area } & \multicolumn{1}{c|}{ Height } & Conc. & \multicolumn{1}{c|}{ Area\% } & Height\% \\
\hline 1 & 3.254 & 63305 & 13320 & 0.000 & 0.256 & 0.721 \\
\hline 2 & 3.582 & 24677309 & 1835155 & 0.000 & 99.744 & 99.279 \\
\hline Total & & 24740615 & 1848476 & & 100.000 & 100.000 \\
\hline
\end{tabular}

